

\title{
Hasse principle for Kummer varieties
}

\author{
Yonatan Harpaz and Alexei N. Skorobogatov
}

\begin{abstract}
The existence of rational points on the Kummer variety associated to a 2-covering of an abelian variety $A$ over a number field can sometimes be established through the variation of the 2-Selmer group of quadratic twists of $A$. In the case when the Galois action on the 2-torsion of $A$ has a large image, we prove, under mild additional hypotheses and assuming the finiteness of relevant Shafarevich-Tate groups, that the Hasse principle holds for the associated Kummer varieties. This provides further evidence for the conjecture that the Brauer-Manin obstruction controls rational points on $\mathrm{K} 3$ surfaces.
\end{abstract}

\section{Introduction}

The principal aim of this paper is to give some evidence in favour of the conjecture that the Brauer-Manin obstruction is the only obstruction to the Hasse principle for rational points on K3 surfaces over number fields; see [Skorobogatov 2009, p. 1859] and [Skorobogatov and Zarhin 2008, p. 484]. Conditionally on the finiteness of relevant Shafarevich-Tate groups we establish the Hasse principle for certain families of Kummer surfaces. These surfaces are quotients of 2-coverings of an abelian surface $A$ by the antipodal involution, where

(a) $A$ is the product of elliptic curves $A=E_{1} \times E_{2}$, or

(b) $A$ is the Jacobian of a curve $C$ of genus 2 with a rational Weierstrass point.

Both cases are treated by the same method which allows us to prove a more general result for the Kummer varieties attached to 2-coverings of an abelian variety $A$ over a number field $k$, provided certain conditions are satisfied. By a 2-covering we understand a torsor $Y$ for $A$ such that the class $[Y] \in \mathrm{H}^{1}(k, A)$ has order at most 2. Thus $Y$ is the twist of $A$ by a 1-cocycle with coefficients in $A$ [2] acting on $A$ by translations. The antipodal involution $\iota_{A}=[-1]: A \rightarrow A$ induces an involution $\iota_{Y}: Y \rightarrow Y$ and we define the Kummer variety $X=\operatorname{Kum}(Y)$ as the minimal desingularisation of $Y / \iota_{Y}$; see Section 6 for details.

In this introduction we explain the results pertaining to cases (a) and (b) above and postpone the statement of a more general theorem until the next section. In case (a)

MSC2010: primary 14G05; secondary 11J95.

Keywords: Kummer varieties, Hasse principle. 
we have the following result, whose proof can be found at the end of Section 2. We denote by $\Delta(f)$ the discriminant of a (not necessarily monic) polynomial $f(x)$; see (3) for the classical formula for $\Delta(f)$ in the case $\operatorname{deg}(f)=4$.

Theorem A. Let $g_{1}(x)$ and $g_{2}(x)$ be irreducible polynomials of degree 4 over a number field $k$, each with the Galois group $S_{4}$. Let $w_{1}$ and $w_{2}$ be distinct primes of $k$ not dividing 6 such that for all $i, j \in\{1,2\}$ the coefficients of $g_{i}(x)$ are integral at $w_{j}$ and $\operatorname{val}_{w_{j}}\left(\Delta\left(g_{i}\right)\right)=\delta_{i j}$. Let $E_{i}$ be the Jacobian of the curve $y^{2}=g_{i}(x)$, where $i=1,2$. For $i=1,2$, assume the finiteness of the 2-primary torsion subgroup of the Shafarevich-Tate group for each quadratic twist of $E_{i}$ whose 2-Selmer group has rank 1. If the Kummer surface with the affine equation

$$
z^{2}=g_{1}(x) g_{2}(y)
$$

is everywhere locally soluble, then it has a Zariski dense set of $k$-points.

We expect that the conditions of Theorem A are in a certain sense 'generic'. To illustrate this, let $\mathbb{Z}[t]_{\mathrm{deg}=4} \subset \mathbb{Z}[t]$ be the set of polynomials of degree 4 ordered by the maximal height of their coefficients. By a theorem of van der Waerden, $100 \%$ of polynomials in $\mathbb{Z}[t]_{\operatorname{deg}=4}$ have the Galois group $S_{4}$ (see [Cohen 1979, Theorem 1] for a statement over an arbitrary number field). By [Hall 2011, Proposition A.2], for $100 \%$ of monic polynomials $g \in \mathbb{Z}[t]_{\operatorname{deg}=4}$ there exists a place $w$ such that $\operatorname{val}_{w}(\Delta(g))=1$. Similar arguments are likely to show that $100 \%$ of pairs $g_{1}, g_{2} \in$ $\mathbb{Z}[t]_{\mathrm{deg}=4}$ satisfy the condition $\operatorname{val}_{w_{j}}\left(\Delta\left(g_{i}\right)\right)=\delta_{i j}$ for some $w_{1}, w_{2}$. The finiteness of the Shafarevich-Tate group is a well-known conjecture, established by M. Bhargava, C. Skinner and W. Zhang [Bhargava et al. 2014, Theorem 2] for a majority of elliptic curves over $\mathbb{Q}$ ordered by naïve height. Note finally that using [Bright et al. 2016, Theorem 1.4] one can show that the Kummer surface (1) is everywhere locally soluble for a positive proportion of pairs $g_{1}, g_{2} \in \mathbb{Z}[t]_{\mathrm{deg}=4}$.

To give an explicit description of our results in case (b) we need to recall the realisation of Kummer surfaces attached to the Jacobian of a genus- 2 curve as smooth complete intersections of three quadrics in $\mathbb{P}_{k}^{5}$. We mostly follow [Skorobogatov 2010, Section 3]; for the classical theory over an algebraically closed field, see [Dolgachev 2012, Chapter 10].

Let $f(x)$ be a separable monic polynomial of degree 5 over a field $k$ of characteristic different from 2 . Let $C$ be the hyperelliptic curve with the affine equation $y^{2}=f(x)$ and let $A$ be the Jacobian of $C$. Let $L$ be the étale $k$-algebra $k[x] /(f(x))$ and let $\theta \in L$ be the image of $x$. The 2-torsion $\operatorname{Gal}(\bar{k} / k)$-module $A$ [2] is isomorphic to $\mathrm{R}_{L / k}\left(\mu_{2}\right) / \mu_{2}$, where $\mathrm{R}_{L / k}$ is the Weil restriction of scalars. Since [ $L: k]$ is odd, $A[2]$ is a direct summand of $\mathrm{R}_{L / k}\left(\mu_{2}\right)$. It follows that the map $\mathrm{H}^{1}\left(k, \mathrm{R}_{L / k}\left(\mu_{2}\right)\right)=L^{*} / L^{* 2} \rightarrow \mathrm{H}^{1}(k, A[2])$ is surjective and induces an isomorphism $\mathrm{H}^{1}(k, A[2])=L^{*} / k^{*} L^{* 2}$. 
Let $\lambda \in L^{*}$. Let $W_{\lambda} \subset \mathrm{R}_{L / k}\left(\mathbb{G}_{m, L}\right)$ be the closed subscheme given by $z^{2}=\lambda$. It is clear that $W_{\lambda}$ is a $k$-torsor for $\mathrm{R}_{L / k}\left(\mu_{2}\right)$ whose class in $\mathrm{H}^{1}\left(k, \mathrm{R}_{L / k}\left(\mu_{2}\right)\right)=L^{*} / L^{* 2}$ is given by $\lambda$. Let $Z_{\lambda}=W_{\lambda} /\{ \pm 1\}$ be the subscheme of $\mathrm{R}_{L / k}\left(\mathbb{G}_{m, L}\right) /\{ \pm 1\}$ given by the same equation. We obtain that $Z_{\lambda}$ is the $k$-torsor for $A[2]$ whose class in $\mathrm{H}^{1}(k, A[2])=L^{*} / k^{*} L^{* 2}$ is defined by $\lambda$.

Now let $Y_{\lambda}=\left(A \times Z_{\lambda}\right) / A[2]$ be the 2-covering of $A$ obtained by twisting $A$ by $Z_{\lambda}$. Then $\operatorname{Kum}\left(Y_{\lambda}\right)$ is the following smooth complete intersection of three quadrics in $\mathbb{P}\left(\mathrm{R}_{L / k}\left(\mathbb{A}_{L}^{1}\right) \times \mathbb{A}_{k}^{1}\right) \simeq \mathbb{P}_{k}^{5}$ :

$$
\operatorname{Tr}_{L / k}\left(\lambda \frac{u^{2}}{f^{\prime}(\theta)}\right)=\operatorname{Tr}_{L / k}\left(\lambda \frac{\theta u^{2}}{f^{\prime}(\theta)}\right)=\operatorname{Tr}_{L / k}\left(\lambda \frac{\theta^{2} u^{2}}{f^{\prime}(\theta)}\right)-\mathrm{N}_{L / k}(\lambda) u_{0}^{2}=0,
$$

where $u$ is an $L$-variable, $u_{0}$ is a $k$-variable, and $f^{\prime}(x)$ is the derivative of $f(x)$ (cf. equations (7) and (8) in [Skorobogatov 2010]). If $\lambda \in k^{*} L^{* 2}$, then an easy change of variable reduces (2) to the same system of equations with $\lambda=1$. As $Y_{1} \cong A$ has a rational point, this case can be excluded for the purpose of establishing the Hasse principle.

Theorem B. Let $f(x)$ be a monic irreducible polynomial of degree 5 over a number field $k$, and let $L=k[x] /(f(x))$. Let $w$ be an odd prime of $k$ such that the coefficients of $f(x)$ are integral at $w$ and $\operatorname{val}_{w}(\Delta(f))=1$. Let $A$ be the Jacobian of the hyperelliptic curve $y^{2}=f(x)$. Assume the finiteness of the 2-primary torsion subgroup of the Shafarevich-Tate group for each quadratic twist of A whose 2-Selmer group has rank 1 . Let $\lambda \in L^{*}$ be such that for some $r \in k^{*}$ the valuation of $\lambda r$ at each completion of $L$ over $w$ is even, but $\lambda \notin k^{*} L^{* 2}$. If the Kummer surface given by (2) is everywhere locally soluble, then it has a Zariski dense set of k-points.

Let $[\lambda] \in \mathrm{H}^{1}(k, A[2])$ be the class defined by $\lambda$. The conditions imposed on $\lambda$ in Theorem $B$ are equivalent to the condition that $[\lambda] \neq 0$ and $[\lambda]$ is unramified at $w$. Equivalently, the $k$-torsor $Z_{\lambda}$ defined above has a $k_{w}^{\text {un }}$-point, where $k_{w}^{\text {un }}$ is the maximal unramified extension of $k_{w}$, but no $k$-point.

Any Kummer surface (2) can be mapped to $\mathbb{P}_{k}^{3}$ by a birational morphism that contracts 16 disjoint rational curves onto singular points. The image of $\operatorname{Kum}\left(Y_{\lambda}\right)$ is a singular quartic surface $S \subset \mathbb{P}_{k}^{3}$ which is the classical Kummer surface with 16 nodes. (See [Dolgachev 2012, Section 10.3.3] and [Gonzalez-Dorrego 1994] for a modern account of the geometry of $S$ over an algebraically closed field.) The group $A$ [2] acts on $S$ by projective automorphisms and the singular locus $S_{\text {sing }}$ is a $k$-torsor for

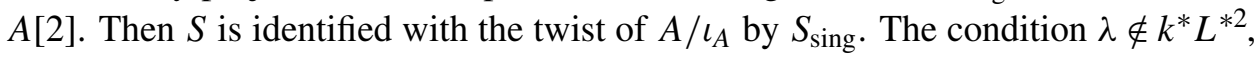
which we use to prove the Zariski density of $S(k)$, is precisely the condition that the torsor $S_{\text {sing }}$ is nontrivial, that is, no singular point of $S$ is a $k$-point.

Theorem B is proved at the end of Section 2. The main idea of the proof of Theorems $\mathrm{A}$ and $\mathrm{B}$ is due to Swinnerton-Dyer. Let $\alpha \in \mathrm{H}^{1}(k, A[2])$ be the class 
of a 1-cocycle used to obtain $Y$ from $A$. The group $\mu_{2}=\{ \pm 1\}$ acts on $A$ by multiplication. As this action commutes with the action of $A[2]$ by translations, we have an induced action of $\mu_{2}$ on $Y$. For an extension $F / k$ of degree at most 2, let $T_{F}$ be the torsor for $\mu_{2}$ defined by $F$. The quadratic twists $A^{F}$ and $Y^{F}$ are defined as the quotients of $A \times_{k} T_{F}$ and $Y \times_{k} T_{F}$, respectively, by the diagonal action of $\mu_{2}$. We identify $A^{F}[2]=A[2]$ and consider $Y^{F}$ as a torsor for $A^{F}$ defined by the same 1-cocycle with the class $\alpha \in \mathrm{H}^{1}\left(k, A^{F}[2]\right)=\mathrm{H}^{1}(k, A[2])$. The projection to the first factor defines a morphism $Y^{F}=\left(Y \times_{k} T_{F}\right) / \mu_{2} \longrightarrow Y / \mu_{2}$. Thus in order to find a rational point on the $\operatorname{Kummer}$ variety $X=\operatorname{Kum}(Y)$ it is enough to find a rational point on $Y^{F}$ for some $F$. At the first step of the proof, using a fibration argument, one produces a quadratic extension $F$ such that $Y^{F}$ is everywhere locally soluble. Equivalently, $\alpha \in \mathrm{H}^{1}\left(k, A^{F}[2]\right)$ is in the 2-Selmer group of $A^{F}$. At the second step one modifies $F$ so that the 2-Selmer group of $A^{F}$ is spanned by $\alpha$ and the image of $A^{F}[2](k)$ under the Kummer map. (In the cases considered in this paper, $A^{F}[2](k)=A[2](k)=0$.) This implies that $\amalg\left(A^{F}\right)[2]$ is $\mathbb{Z} / 2$ or 0 . In previous applications of the method [Swinnerton-Dyer 2001; Skorobogatov and Swinnerton-Dyer 2005], as well as in Theorem A above, $A$ is a product of two elliptic curves, in which case the Cassels-Tate pairing on $\amalg\left(A^{F}\right)$ is alternating. The assumption that $\amalg\left(A^{F}\right)$ is finite then implies that the order of $\amalg\left(A^{F}\right)[2]$ is a square and hence $\amalg\left(A^{F}\right)[2]=0$. In particular, $Y^{F}$ has a $k$-point, so that $Y^{F} \simeq A^{F}$. In this paper we consider more general principally polarised abelian varieties. The theory developed by Poonen and Stoll [1999] ensures that in the cases considered here the Cassels-Tate pairing on $\amalg\left(A^{F}\right)$ defined using the principal polarisation is still alternating, so the proof can be concluded as before.

Swinnerton-Dyer's method was used in combination with Schinzel's Hypothesis (H) in [Colliot-Thélène et al. 1998; Swinnerton-Dyer 2000; Wittenberg 2007]. It is in [Swinnerton-Dyer 2001] that the method was applied without Hypothesis (H) for the first time, using Dirichlet's theorem on primes in an arithmetic progression, the only known case of $(\mathrm{H})$. That work tackled diagonal cubic surfaces, which are dominated by a product of two elliptic curves with complex multiplication. The immediate precursor of our Theorem A is [Skorobogatov and Swinnerton-Dyer 2005], which treats Kummer surfaces attached to products of elliptic curves, again without assuming Hypothesis $(\mathrm{H})$. Central to Swinnerton-Dyer's method is a linear algebra construction that represents the Selmer group as the kernel of a symmetric bilinear form. The difficulty of operating this machinery makes implementation of the method a rather delicate task. In the present paper this linear algebra machinery is not used. Instead we use the ideas from [Mazur and Rubin 2007] and especially from [Mazur and Rubin 2010].

Let us note that given an elliptic curve $E$ over a number field $k$ it is not always possible to find a quadratic extension $F / k$ such that the 2-Selmer group of $E^{F}$ is 
spanned by a fixed class $\alpha \in \mathrm{H}^{1}(k, E[2])$ and the image of $E^{F}[2](k)$. Firstly, the parity of the rank of the 2-Selmer group of $E^{F}$ can be the same for all $F$ : this happens precisely when $k$ is totally imaginary and $E$ acquires everywhere good reduction over an abelian extension of $k$; see [Dokchitser and Dokchitser 2011, Remark 4.9]. Secondly, over any number field $k$ there are elliptic curves $E$ such that, for any quadratic extension $F / k$, the difference between the 2-Selmer rank of $E^{F}$ and the dimension of the $\mathbb{F}_{2}$-vector space $E[2](k)$ is at least the number of complex places of $k$; see [Klagsbrun 2012a; 2012b]. Such examples can occur when $E[2](k) \cong \mathbb{Z} / 2$ and $E$ has a cyclic isogeny of degree 4 defined over $k(E[2])$ but not over $k$.

In this paper we do not discuss the conjecture (for which see [Skorobogatov 2009, p. 1859; Skorobogatov and Zarhin 2008, p. 484]) that rational points on a K3 surface are dense in its Brauer-Manin set. ${ }^{1}$ Nevertheless we make the following simple observation in the direction of Mazur's conjectures [1992; 1995]:

Proposition 1.1. Let $E_{1}, \ldots, E_{n}$ be elliptic curves over $\mathbb{Q}$ such that $E_{i}[2](\mathbb{Q})=0$ for $i=1, \ldots, n$. Let $X=\operatorname{Kum}\left(\prod_{i=1}^{n} Y_{i}\right)$, where $Y_{i}$ is a 2-covering of $E_{i}$ defined by a class in $\mathrm{H}^{1}\left(\mathbb{Q}, E_{i}[2]\right)$ that restricts to a nonzero class in $\mathrm{H}^{1}\left(\mathbb{R}, E_{i}[2]\right)$, for $i=1, \ldots, n$. Then the real topological closure of $X(\mathbb{Q})$ in $X(\mathbb{R})$ is a union of connected components of $X(\mathbb{R})$.

This can be compared with the result of M. Kuwata and L. Wang [1993]. See the end of Section 7 for the proof of Proposition 1.1.

Our main technical result is Theorem 2.3. It is stated in Section 2 where we also show that Theorem 2.3 implies Theorems A and B. In Section 3 we systematically develop the Galois-theoretic aspect of the approach of Mazur and Rubin. We recall the necessary facts about the Kummer map for quadratic twists of abelian varieties over local fields in Section 4. In Section 5 we discuss the Selmer group and the Cassels-Tate pairing over a number field. A reduction to everywhere soluble 2-coverings is carried out in Section 6 using a known case of the fibration method. We finish the proof of Theorem 2.3 in Section 7.

\section{Main results}

Let $k$ be a field of characteristic different from 2 with a separable closure $\bar{k}$ and the Galois group $\Gamma_{k}=\operatorname{Gal}(\bar{k} / k)$.

Let $A$ be an abelian variety over $k$. Let $K=k(A[2]) \subset \bar{k}$ be the field of definition of $A[2]$, that is, the smallest field such that $A[2](K)=A[2](\bar{k})$. Let $G=\operatorname{Gal}(K / k)$. Consider the following conditions:

${ }^{1}$ A recent result of D. Holmes and R. Pannekoek [2015] shows that if this conjecture is extended to all Kummer varieties, then the ranks of quadratic twists of any given abelian variety over a given number field are not bounded. 
(a) $A[2]$ is a simple $G$-module and $\operatorname{End}_{G}(A[2])=\mathbb{F}_{2}$;

(b) $\mathrm{H}^{1}(G, A[2])=0$;

(c) there exists a $g \in G$ such that $A[2] /(g-1)=\mathbb{F}_{2}$;

(d) there exists an $h \in G$ such that $A[2] /(h-1)=0$.

Lemma 2.1. Let $A$ be the Jacobian of a smooth projective curve with the affine equation $y^{2}=f(x)$, where $f(x) \in k[x]$ is an irreducible separable polynomial of odd degree $m \geq 3$. If the Galois group of $f(x)$ is the symmetric group $S_{m}$ on $m$ letters, then A satisfies conditions (a), (b), (c), (d).

Proof. It is well-known that the $\Gamma_{k}$-module $A[2]$ is the zero-sum submodule of the vector space $\left(\mathbb{F}_{2}\right)^{m}$ freely generated by the roots of $f(x)=0$ with the natural permutation action of $\Gamma_{k}$. Since $m$ is odd, the permutation $\Gamma_{k}$-module $\left(\mathbb{F}_{2}\right)^{m}$ is the direct sum of $A[2]$ and the $\mathbb{F}_{2}$-vector space spanned by the vector $(1, \ldots, 1)$.

If an $S_{m}$-submodule of $\left(\mathbb{F}_{2}\right)^{m}$ contains a vector with at least one coordinate 0 and at least one coordinate 1 , then it contains the zero-sum submodule. Hence $A[2]$ is a simple $S_{m}$-module. A direct calculation with matrices shows that the $m \times m$ matrices commuting with all permutation matrices are the linear combinations of the identity and the all-1 matrix. We deduce that $\operatorname{End}_{S_{m}}(A[2])=\mathbb{F}_{2}$, thus (a) holds.

The permutation $S_{m}$-module $\left(\mathbb{F}_{2}\right)^{m}$ is isomorphic to $\mathbb{F}_{2}\left[S_{m} / S_{m-1}\right]$. By Shapiro's lemma we have

$$
\mathrm{H}^{1}\left(S_{m}, \mathbb{F}_{2}\left[S_{m} / S_{m-1}\right]\right)=\mathrm{H}^{1}\left(S_{m-1}, \mathbb{F}_{2}\right)=\operatorname{Hom}\left(S_{m-1}, \mathbb{F}_{2}\right)=\mathbb{F}_{2} .
$$

Since $\mathrm{H}^{1}\left(S_{m}, \mathbb{F}_{2}\right)=\mathbb{F}_{2}$, we obtain $\mathrm{H}^{1}\left(S_{m}, A[2]\right)=0$, so (b) holds.

If $g$ is a cycle of length $m-1$, then $A[2] /(g-1)=\mathbb{F}_{2}$, so (c) is satisfied. If $h$ is a cycle of length $m$, then $A[2] /(h-1)=0$, so (d) is satisfied.

Remark 2.2. There are other natural cases when the Galois module $A[2]$ satisfies conditions (a) through (d). Let $\operatorname{dim}(A)=n>1$. We only deal with the case when the Cassels-Tate pairing on $\amalg(A)$ defined by a polarisation $\lambda \in \mathrm{NS}(\bar{A})^{\Gamma_{k}}$ is alternating. According to the results of Poonen, Stoll and Rains recalled in Section 5, this holds when $\lambda$ lifts to a symmetric element of $\operatorname{Pic}(A)$. (This happens, for example, when $A$ is as in Lemma 2.1.) In this case the pairing $A[2] \times A[2] \rightarrow \mathbb{Z} / 2$ induced by $\lambda$ and the Weil pairing admits a Galois-invariant quadratic refinement $q: A[2] \rightarrow \mathbb{Z} / 2$; see [Poonen and Rains 2011, Proposition 3.2(c)]. The 'generic' Galois action compatible with this assumption is when $G$ is the corresponding orthogonal group $O(q) \subset \mathrm{GL}(A[2])$. It can be shown that conditions (a), (c) and (d) are always satisfied for $G=O(q)$. Condition (b) is satisfied for all $n \neq 2,3$ when $q$ is split (i.e., isomorphic to a direct sum of copies of the rank-2 hyperbolic space) and for all $n \neq 3,4$ if $q$ is nonsplit (see [Sah 1977, Proposition 2.1]). We do not elaborate on these statements here, as we will not use them. 
Let $A_{1}, \ldots, A_{r}$ be abelian varieties over $k$. For each $i=1, \ldots, r$, let $K_{i}=k\left(A_{i}[2]\right)$ and $G_{i}=\operatorname{Gal}\left(K_{i} / k\right)$. We assume the following condition:

(e) The fields $K_{1}, \ldots, K_{r}$ are linearly disjoint over $k$.

By definition this means that $\left[K_{1} \cdots K_{r}: k\right]=\prod_{i=1}^{r}\left[K_{i}: k\right]$. Thus the Galois group of $K_{1} \cdots K_{r}$ over $k$ is $\prod_{i=1}^{r} G_{i}$.

When $k$ is a number field we shall also assume the following condition:

(f) There exist distinct odd primes $w_{1}, \ldots, w_{r}$ of $k$ such that for each $i=1, \ldots, r$ the abelian variety $A_{i}$ has bad reduction at $w_{i}$ and the number of geometric connected components of the Néron model of $A_{i}$ at $w_{i}$ is odd, whereas each $A_{j}$ for $j \neq i$ has good reduction at $w_{i}$.

Let $k_{i}^{\mathrm{ab}}$ be the maximal abelian subextension of $k \subset K_{i}$. Equivalently, $\operatorname{Gal}\left(k_{i}^{\mathrm{ab}} / k\right)$ is the maximal abelian quotient $G_{i}^{\mathrm{ab}}$ of $G_{i}$. Let us finally assume:

(g) For each $i=1, \ldots, r$, the field $k_{i}^{\mathrm{ab}}$ is totally ramified at $w_{i}$. Equivalently, $k_{i}^{\mathrm{ab}}$ has a unique prime ideal above $w_{i}$, and $G_{i}^{\text {ab }}$ coincides with the inertia subgroup of this ideal.

Let $F$ be a field extension of $k$ of degree at most 2. As in the introduction, we denote by $A^{F}$ the quadratic twist of $A$ by $F$, that is, the abelian variety over $k$ obtained by twisting $A$ by the quadratic character of $F / k$ with respect to the action of $\mu_{2}$ on $A$ by multiplication. For example, if $A$ is an elliptic curve with the Weierstrass equation $y^{2}=f(x)$, then $A^{F}$ is given by $y^{2}=c f(x)$, where $c \in k^{*}$ is such that $F=k(\sqrt{c})$.

We are now ready to state the main theorem. Recall that a class in $\mathrm{H}^{1}(k, A[2])$ is said to be unramified at an odd non-Archimedean place $v$ of $k$ if it goes to zero under the restriction map $\mathrm{H}^{1}(k, A[2]) \rightarrow \mathrm{H}^{1}\left(k_{v}^{\mathrm{nr}}, A[2]\right)$, where $k_{v}^{\mathrm{nr}}$ is the maximal unramified extension of the completion $k_{v}$ of $k$ at $v$.

Theorem 2.3. Let $k$ be a number field. Let $A=\prod_{i=1}^{r} A_{i}$, where each $A_{i}$ is a principally polarised abelian variety satisfying conditions (a), (b), (c) and (d). Assume in addition that conditions (e), (f) and (g) are satisfied. Assume that the 2-primary subgroup of the Shafarevich-Tate group $\amalg\left(A_{i}^{F}\right)\{2\}$ is finite for all $i=1, \ldots, r$ and all extensions $F$ of $k$ with $[F: k] \leq 2$ for which the 2-Selmer group of $A_{i}^{F}$ has rank 1 . Consider the classes in $\mathrm{H}^{1}(k, A[2])$ that are unramified at $w_{1}, \ldots, w_{r}$ and whose projection to $\mathrm{H}^{1}\left(k, A_{i}[2]\right)$ is nonzero for each $i=1, \ldots, r$. If the Kummer variety of A defined by such a class is everywhere locally soluble, then it has a Zariski dense set of k-points.

Remarks. (1) If $r=1$, then condition (d) is not needed and condition (e) is vacuous.

(2) The Brauer-Manin obstruction does not appear in the conclusion of the theorem. In fact, the purely algebraic conditions (a), (b) and (e) imply that a certain part of 
the Brauer group is trivial; see Proposition 6.1. The problem of calculation of the full Brauer group of a Kummer variety will be addressed in a separate paper.

(3) If the 2-primary torsion subgroup $\amalg\left(A_{i}^{F}\right)\{2\}$ is finite, then condition (b) implies that the nondegenerate Cassels-Tate pairing on $\amalg\left(A_{i}^{F}\right)\{2\}$ is alternating. See Proposition 5.2, which is based on the work of [Poonen and Stoll 1999] and [Poonen and Rains 2011]. In the proof of Theorem 2.3 we use a well-known consequence of this result that the number of elements of $\amalg\left(A_{i}^{F}\right)[2]$ is a square.

We employ the following standard notation:

- $k_{w_{i}}$ is the completion of $k$ at $w_{i}$,

- $\mathcal{O}_{w_{i}}$ is the ring of integers of $k_{w_{i}}$,

- $\mathfrak{m}_{w_{i}}$ is the maximal ideal of $\mathcal{O}_{w_{i}}$, and

- $\mathbb{F}_{w_{i}}=\mathcal{O}_{w_{i}} / \mathfrak{m}_{w_{i}}$ is the residue field.

Corollary 2.4. Let $k$ be a number field. For $i=1, \ldots, r$, let $f_{i}(x) \in k[x]$ be a monic irreducible polynomial of odd degree $n_{i} \geq 3$ whose Galois group is the symmetric group $S_{n_{i}}$, and let $A_{i}$ be the Jacobian of the hyperelliptic curve $y^{2}=f_{i}(x)$. Assume the existence of distinct odd primes $w_{1}, \ldots, w_{r}$ of $k$ such that $f_{i}(x) \in \mathcal{O}_{w_{j}}[x]$ and $\operatorname{val}_{w_{i}}\left(\Delta\left(f_{j}\right)\right)=\delta_{i j}$ for any $i, j \in\{1, \ldots, r\}$. Assume that $\amalg\left(A_{i}^{F}\right)\{2\}$ is finite for all $i=1, \ldots, r$ and all extensions $F$ of $k$ with $[F: k] \leq 2$ for which the 2-Selmer group of $A_{i}^{F}$ has rank 1 . Consider the classes in $\mathrm{H}^{1}(k, A[2])$ that are unramified at $w_{1}, \ldots, w_{r}$ and whose projection to $\mathrm{H}^{1}\left(k, A_{i}[2]\right)$ is nonzero for each $i=1, \ldots, r$. If the Kummer variety of $A$ defined by such a class is everywhere locally soluble, then it has a Zariski dense set of k-points.

Proof. Each $A_{i}$ is a canonically principally polarised abelian variety which satisfies conditions (a) through (d) by Lemma 2.1.

Let $C_{i}$ be the proper, smooth and geometrically integral curve over $k$ given by the affine equation $y^{2}=f_{i}(x)$, so that $A_{i}=\operatorname{Jac}\left(C_{i}\right)$. As in [Liu 1996, Section 4.3], a proper and flat Weierstrass model $\mathcal{C}_{i}$ over $\operatorname{Spec}\left(\mathcal{O}_{w_{i}}\right)$ is defined as the normalisation in $C_{i} \times_{k} k_{w_{i}}$ of the projective line $\mathbb{P}_{\mathcal{O}_{w_{i}}}^{1}$ with the affine coordinate $x$. Since $2 \in \mathcal{O}_{w_{i}}^{*}$, the integral closure of $\mathcal{O}_{w_{i}}[x]$ in $k_{w_{i}}\left(C_{i}\right)$ is $\mathcal{O}_{w_{i}}[x, y] /\left(y^{2}-f_{i}(x)\right)$. The condition $\operatorname{val}_{w_{i}}\left(\Delta\left(f_{i}\right)\right)=1$ implies that $\mathcal{C}_{i}$ is regular and the special fibre $\mathcal{C}_{i} \times \mathcal{O}_{w_{i}} \mathbb{F}_{w_{i}}$ is geometrically integral with a unique singular point, which is an ordinary double point; see Corollary 6 and Remark 18 on p. 4602 of [Liu 1996]. In particular, the reduction of $f_{i}(x)$ modulo $\mathfrak{m}_{w_{i}}$ has one rational double root and $n_{i}-2$ simple roots. (This can also be checked directly using Sylvester's formula for the discriminant.) Now [Bosch et al. 1990, Theorem 9.6.1] implies that the special fibre of the Néron model of $A_{i} \times_{k} k_{w_{i}}$ over $\operatorname{Spec}\left(\mathcal{O}_{w_{i}}\right)$ is connected. If $j \neq i$, then $\operatorname{val}_{w_{i}}\left(\Delta\left(f_{j}\right)\right)=0$, and this implies that $A_{j}$ has good reduction at $w_{i}$. We conclude that (f) holds. 
For each $i=1, \ldots, r$, the field $K_{i}=k\left(A_{i}[2]\right)$ is the splitting field of $f_{i}(x)$. Since $\operatorname{Gal}\left(K_{i} / k\right) \cong S_{n_{i}}$, the alternating group is the unique nontrivial normal subgroup of $\operatorname{Gal}\left(K_{i} / k\right)$. Its invariant subfield is $k\left(\sqrt{\Delta\left(f_{i}\right)}\right)$. Thus if $k^{\prime}$ is a Galois extension of $k$ such that $k \subsetneq k^{\prime} \subsetneq K_{i}$, then $k^{\prime}=k\left(\sqrt{\Delta\left(f_{i}\right)}\right)=k_{i}^{\mathrm{ab}}$. The extension $k\left(\sqrt{\Delta\left(f_{i}\right)}\right)$ of $k$ is ramified at $w_{i}$, so (g) holds.

Let $K_{i}^{\prime}$ be the compositum of the fields $K_{j}$ for $j \neq i$. Since each $K_{i}$ is a Galois extension of $k$, the field $K_{i} \cap K_{i}^{\prime}$ is also a Galois extension of $k$. To verify (e) we need to check that $K_{i} \cap K_{i}^{\prime}=k$ for each $i=1, \ldots, r$. Otherwise, $K_{i} \cap K_{i}^{\prime}$ contains $k\left(\sqrt{\Delta\left(f_{i}\right)}\right)$ which is ramified at $w_{i}$. However, this contradicts the criterion of NéronOgg-Shafarevich according to which $K_{i}^{\prime}$ is unramified at the odd place $w_{i}$, where each of the abelian varieties $A_{j}$ for $j \neq i$ has good reduction. Thus (e) holds.

Proof of Theorem A assuming Theorem 2.3. For $i=1,2$, let $C_{i}$ be the curve of genus 1 given by $y^{2}=g_{i}(x)$. Write $g_{i}(x)=a x^{4}+b x^{3}+c x^{2}+d x+e$. The classical $\mathrm{SL}(2)$-invariants of the corresponding quartic binary form $G_{i}(u, v)=v^{4} g_{i}(u / v)$ are

$$
\begin{aligned}
I & =12 a e-3 b d+c^{2}, \\
J & =72 a c e+9 b c d-27 a d^{2}-27 e b^{2}-2 c^{3}, \\
\Delta & =\left(4 I^{3}-J^{2}\right) / 27 .
\end{aligned}
$$

Then the Jacobian of $C_{i}$ is the elliptic curve $E_{i}$ with the equation $u^{2}=p_{i}(t)$, where $p_{i}(t)=t^{3}-27 I x-27 \mathrm{~J}$ is the resolvent cubic polynomial of $g_{i}(x)$; see [Skorobogatov 2001, Proposition 3.3.6(a)]. The 0-dimensional scheme $g_{i}(x)=0$ is a $k$-torsor $Z_{i}$ for $E_{i}$ [2]. Then $C_{i}$ can be viewed as the twist of $E_{i}$ by $Z_{i}$; that is, $C_{i}=\left(E_{i} \times Z_{i}\right) / E_{i}$ [2], where $E_{i}$ [2] acts simultaneously on both factors. The antipodal involution acts on $C_{i}$ by changing the sign of $y$, so the Kummer surface $\operatorname{Kum}\left(C_{1} \times C_{2}\right)$ is the minimal desingularisation of the quotient of $C_{1} \times C_{2}$ by the involution that acts on each component as $(x, y) \mapsto(x,-y)$. Thus $z^{2}=g_{1}(x) g_{2}(y)$ defines an affine surface birationally equivalent to $\operatorname{Kum}\left(C_{1} \times C_{2}\right)$.

Since the polynomials $g_{1}(x)$ and $g_{2}(x)$ have no roots in $k$, each of the torsors $Z_{1}$ and $Z_{2}$ is nontrivial. The field of definition $K_{i}=k\left(E_{i}[2]\right)$ of $E_{i}$ [2] is the splitting field of $p_{i}(t)$. Hence the condition $\operatorname{Gal}\left(g_{1}\right) \simeq S_{4}$ implies $\operatorname{Gal}\left(K_{i} / k\right)=\operatorname{Gal}\left(p_{i}\right) \simeq S_{3}$, for $i=1,2$. The discriminant of the quartic $g_{i}(x)$ is equal to the discriminant of its resolvent cubic $p_{i}(t)$ up to a power of 3 , and $g_{i}(x) \in \mathcal{O}_{w_{j}}[x]$ implies $p_{i}(t) \in \mathcal{O}_{w_{j}}[t]$, so the primes $w_{1}$ and $w_{2}$ satisfy the assumption in Corollary 2.4. To be in a position to appeal to that corollary we now show that $Z_{i}$ is unramified at both $w_{1}$ and $w_{2}$.

Indeed, let $\mathcal{Z}_{i j} \subset \mathbb{P}_{\mathcal{O}_{w_{j}}}^{1}$ be the closed subscheme given by $G_{i}(u, v)=0$, where $G_{i}(u, v)=v^{4} g_{i}(u / v)$. For $j \neq i$, the discriminant of $G_{i}(u, v)$ is a unit in $\mathcal{O}_{w_{j}}$. Thus $\mathcal{Z}_{i j}$ is a finite and étale $\mathcal{O}_{w_{j}}$-scheme of degree 4 with the generic fibre $Z_{i} \times_{k} k_{w_{j}}$, hence $Z_{i}$ is unramified at $w_{j}$. For $i=j$, the discriminant of $G_{i}(u, v)$ is a generator 
of the maximal ideal of $\mathcal{O}_{w_{i}}$. This implies that the fibre $\mathcal{Z}_{i i} \times_{\mathcal{O}_{w_{i}}} \mathbb{F}_{w_{i}}$ at the closed point of $\operatorname{Spec}\left(\mathcal{O}_{w_{i}}\right)$ is the disjoint union of a double $\mathbb{F}_{w_{i}}$-point and a reduced 2-point $\mathbb{F}_{w_{i}}$-scheme. The latter gives rise to two sections of the morphism

$$
\mathcal{Z}_{i i} \times \mathcal{O}_{w_{i}} \mathcal{O}_{w_{i}}^{\mathrm{nr}} \longrightarrow \operatorname{Spec}\left(\mathcal{O}_{w_{i}}^{\mathrm{nr}}\right)
$$

Hence $Z_{i}$ is unramified at $w_{i}$. An application of Corollary 2.4 finishes the proof.

Proof of Theorem B assuming Theorem 2.3. The condition $\operatorname{val}_{w}(\Delta(f))=1$ implies that $k(\sqrt{\Delta(f)})$ has degree 2 over $k$. Hence the Galois group of $f(x)$ is not a subgroup of the alternating group $A_{5}$. Any proper subgroup of $S_{5}$ which acts transitively on $\{1,2,3,4,5\}$ and is not contained in $A_{5}$ is conjugate to $\operatorname{Aff}_{5}=\mathbb{F}_{5} \rtimes \mathbb{F}_{5}^{*}$, the group of affine transformations of the affine line over the finite field $\mathbb{F}_{5}$; see [Burnside 1911, Chapter XI, §166, p. 215]. Let us show that this case cannot occur. Indeed, in the proof of Corollary 2.4 we have seen that the reduction of $f(x)$ modulo $\mathfrak{m}_{w}$ has one rational double root and three simple roots, whereas the integral model defined by $y^{2}=f(x)$ is regular. It follows that over the maximal unramified extension of $k_{w}$ the polynomial $f(x)$ is the product of three linear and one irreducible quadratic polynomials. Hence the image of the inertia subgroup in Aff $_{5}$ is generated by a cycle of length 2 . This is a contradiction because the elements of order 2 in Aff $_{5}$ are always products of two cycles, as they are given by affine transformations of the form $x \mapsto-x+a$.

We conclude that the Galois group of $f(x)$ is $S_{5}$. The theorem now follows from Corollary 2.4, provided we check that the relevant class in $\mathrm{H}^{1}(k, A[2])$ is nonzero and unramified at $w$.

For this it is enough to prove that the corresponding $k$-torsor for $A$ [2] has no $k$-points but has a $k_{w}^{\text {un }}$-point. This torsor is the subset $Z_{\lambda} \subset \mathrm{R}_{L / k}\left(\mathbb{G}_{m, L}\right) /\{ \pm 1\}$ given by $z^{2}=\lambda$. The natural surjective map

$$
\mathrm{R}_{L / k}\left(\mathbb{G}_{m, L}\right) \longrightarrow \mathrm{R}_{L / k}\left(\mathbb{G}_{m, L}\right) /\{ \pm 1\}
$$

is a torsor for $\mu_{2}$. Thus $Z_{\lambda}(k)$ is the disjoint union of the images of $k$-points of the torsors $t z^{2}=\lambda$ for $\mathrm{R}_{L / k}\left(\mu_{2}\right)$, where $t \in k^{*}$. Hence $Z_{\lambda}(k) \neq \varnothing$ if and only if $\lambda \in k^{*} L^{* 2}$, but this is excluded by one of the assumptions of Theorem B. Next, the group $\mathrm{H}^{1}\left(k_{w}^{\mathrm{un}}, \mu_{2}\right)$ consists of the classes of 1-cocycles defined by 1 and $\pi$, where $\pi$ is a generator of $\mathfrak{m}_{w}$. Hence $Z_{\lambda}\left(k_{w}^{\text {un }}\right)$ is the disjoint union of the images of $k_{w}^{\text {un }}$-points of the torsors $z^{2}=\lambda$ and $z^{2}=\pi \lambda$ for $\mathrm{R}_{L / k}\left(\mu_{2}\right)$. By assumption there exists an $\varepsilon \in\{0,1\}$ such that the valuation of $\pi^{\varepsilon} \lambda$ at each completion of $L$ over $w$ is even. Then the torsor for $\mathrm{R}_{L / k}\left(\mu_{2}\right)$ given by $z^{2}=\pi^{\varepsilon} \lambda$ has a $k_{w}^{\text {un }}$-point, because any unit is a square, as the residue field of $k_{w}^{\text {un }}$ is separably closed and of characteristic different from 2. It follows that $Z_{\lambda}\left(k_{w}^{\text {un }}\right) \neq \varnothing$. 


\section{Galois theory of finite torsors}

This section develops some ideas from [Mazur and Rubin 2010]; see Lemma 3.5 in particular.

We shall work with groups that are semidirect products of a group $G$ with a semisimple $G$-module $M$. Recall that a $G$-module $M$ is simple if it has no $G$-submodules except 0 and $M$. A $G$-module $M$ is semisimple if $M$ is a direct sum of simple $G$-modules $M=\bigoplus_{i} M_{i}$. The simple $G$-modules $M_{i}$ are called the simple factors of $M$. Their isomorphism types do not depend on the presentation of $M$ as a direct sum. Indeed, one can characterise the simple factors of $M$ as the simple $G$-modules that admit a nonzero map to $M$ or from $M$.

Remark 3.1. If $M$ is a semisimple $G$-module, then each $G$-submodule of $M$ is a direct summand of $M$; see, e.g., [Wisbauer 1991, 20.2]. Furthermore, each $G$-submodule $N \subseteq M$ is semisimple and each simple factor of $N$ is a simple factor of $M$. Similarly, each quotient $G$-module $M / N$ is semisimple and each simple factor of $M / N$ is a simple factor of $M$.

Lemma 3.2. Let $G$ be a group with more than one element and let $M$ be a semisimple $G$-module such that the action of $G$ on each simple factor of $M$ is faithful. Let $H \subseteq M \rtimes G$ be a normal subgroup. Then

(i) either $H \subseteq M$ or $M \subseteq H$;

(ii) if $(M \rtimes G) / H$ is abelian, then $M \subseteq H$.

Proof. (i) Suppose that $M$ is not contained in $H$. The subgroup $K=H \cap M$ is normal in $M \rtimes G$, thus $K$ is a proper $G$-submodule of $M$. The quotient $G$-module $N=M / K \neq 0$ is semisimple by Remark 3.1. Moreover, each simple factor of $N$ is a simple factor of $M$, hence $N$ is a faithful $G$-module. We identify $K$ with the kernel of the natural surjective group homomorphism $\rho: M \rtimes G \rightarrow N \rtimes G$. Then $\rho(H)$ and $N$ are normal subgroups of $N \rtimes G$ such that $\rho(H) \cap N=\{1\}$, hence they centralise each other. Thus the image of $H$ in $G$ acts trivially on $N$. But $N$ is a faithful $G$-module, so the image of $H$ in $G$ is trivial, hence $H \subseteq M$.

(ii) By the result of (i) we just need to show that the case $H \subsetneq M$ is not possible. Indeed, since $H$ is normal in $M \rtimes G$, in this case $H$ is a proper $G$-submodule of $M$, so that $(M \rtimes G) / H=N \rtimes G$, where $N=M / H \neq 0$. The same argument as in the proof of (i) shows that $N$ is a faithful $G$-module. By assumption $(M \rtimes G) / H$ is abelian, so $G$ acts trivially on $N$. This contradicts the fact that $G$ contains an element other than the unit of the group law.

Let us now set up notation and terminology for this section.

Let $k$ be a field and $\bar{k}$ a separable closure of $k$, and let $\Gamma_{k}=\operatorname{Gal}(\bar{k} / k)$. Let $M$ be a finite $\Gamma_{k}$-module such that the order of $M$ is not divisible by char $(k)$. We denote by 
$\varphi: \Gamma_{k} \rightarrow \operatorname{Aut}(M)$ the action of $\Gamma_{k}$ on $M$. We identify $M$ with the group of $\bar{k}$-points of a finite étale commutative group $k$-scheme $\mathcal{G}_{M}$. A cocycle $c: \Gamma_{k} \rightarrow M=\mathcal{G}_{M}(\bar{k})$ gives rise to a twisted action of $\Gamma_{k}$ on $\mathcal{G}_{M}(\bar{k})$, defined as the original action of $\Gamma_{k}$ on $M$ followed by the translation by $c$. The quotient of $\operatorname{Spec}\left(\bar{k}\left[\mathcal{G}_{M}\right]\right)$ by the twisted action is a $k$-torsor of $\mathcal{G}_{M}$. It comes equipped with a $\bar{k}$-point corresponding to the neutral element of $\mathcal{G}_{M}$. Conversely, suppose we are given a $k$-torsor $Z$ for $\mathcal{G}_{M}$. For any $z_{0} \in Z(\bar{k})$, the map $c: \Gamma_{k} \rightarrow M=\mathcal{G}_{M}(\bar{k})$ determined by the condition $c(\gamma) z_{0}=\gamma_{z_{0}}$ is a cocycle $\Gamma_{k} \rightarrow M$. These constructions induce a bijection between $\mathrm{H}^{1}(k, M)$ and the set of isomorphisms classes of $k$-torsors for $\mathcal{G}_{M}$. See [Skorobogatov 2001, Section 2.1], and also [Bosch et al. 1990, Chapter 6]. For $\alpha \in \mathrm{H}^{1}(k, M)$, we denote by $Z_{\alpha}$ the torsor for $\mathcal{G}_{M}$ obtained by twisting $\mathcal{G}_{M}$ by a 1-cocycle representing $\alpha$; such a torsor is well-defined up to an isomorphism of $\mathcal{G}_{M}$-torsors.

Definition 3.3. Let $K$ be the smallest extension of $k$ such that $\Gamma_{K}$ acts trivially on $M$. For $\alpha \in \mathrm{H}^{1}(k, M)$, let $K_{\alpha}$ be the smallest extension of $k$ such that $\Gamma_{K_{\alpha}}$ acts trivially on $Z_{\alpha}(\bar{k})$. Write $G=\operatorname{Gal}(K / k)$ and $G_{\alpha}=\operatorname{Gal}\left(K_{\alpha} / k\right)$.

Note that $K \subset K_{\alpha}$, which follows from the surjectivity of the difference map $Z_{\alpha} \times Z_{\alpha} \rightarrow \mathcal{G}_{M}$. Write $W_{\alpha}=\operatorname{Gal}\left(K_{\alpha} / K\right)$. Then there is an exact sequence

$$
1 \longrightarrow W_{\alpha} \longrightarrow G_{\alpha} \stackrel{\varphi}{\longrightarrow} G \longrightarrow 1 \text {. }
$$

The group $G$ of Definition 3.3 is identified with $\varphi\left(\Gamma_{k}\right)$, which makes $M$ a faithful $G$-module. Let $\alpha \in \mathrm{H}^{1}(k, M)$ be a class represented by a 1-cocycle $c: \Gamma_{k} \rightarrow M$. If $Z_{\alpha}$ is the twist of $\mathcal{G}_{M}$ by $c$, then the semidirect product $M \rtimes G$ acts on $Z_{\alpha}(\bar{k}) \cong$ $\mathcal{G}_{M}(\bar{k}) \cong M$ by affine transformations, and $\Gamma_{k}$ acts on $Z_{\alpha}(\bar{k})$ by the homomorphism $(c, \varphi): \Gamma_{k} \rightarrow M \rtimes G$. By the definition of $K_{\alpha}$ this homomorphism factors through an injective homomorphism $G_{\alpha} \rightarrow M \rtimes G$. Since $M$ is a trivial $\Gamma_{K}$-module, the restriction of $\alpha$ to $W_{\alpha}$ defines an injective homomorphism of $G$-modules $\tilde{\alpha}: W_{\alpha} \rightarrow M$, and we have the commutative diagram

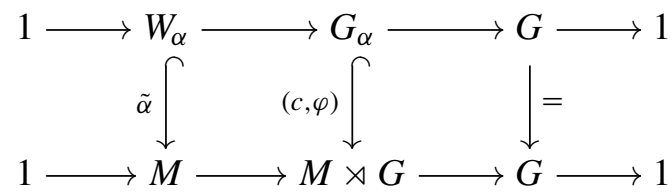

Let $R=\operatorname{End}_{G}(M)=\operatorname{End}_{\Gamma_{k}}(M)$ be the endomorphism ring of the $\Gamma_{k}$-module $M$.

Definition 3.4. Let $N$ be an $R$-module. We say that $\alpha \in N$ is nondegenerate if the annihilator of $\alpha$ in $R$ is zero, i.e., if, for $r \in R, r \alpha=0$ implies $r=0$. Equivalently, $\alpha$ is nondegenerate if $R \alpha \subset N$ is a free $R$-module.

Remark 3.5. For any $\Gamma_{k}$-module $M$, the group $\mathrm{H}^{1}(k, M)$ is naturally an $R$-module. If $M$ is a simple $G$-module, then $R$ is a division ring by Schur's lemma, hence a finite 
field by Wedderburn's theorem. Then an element $\alpha \in \mathrm{H}^{1}(k, M)$ is nondegenerate if and only if $\alpha \neq 0$. When $M=\bigoplus_{i=1}^{r} M_{i}$, where the $G$-modules $M_{i}$ are simple and pairwise nonisomorphic, $R=\bigoplus_{i=1}^{r} \operatorname{End}_{G}\left(M_{i}\right)$ is a direct sum of fields. We have $\mathrm{H}^{1}(k, M)=\bigoplus_{i=1}^{r} \mathrm{H}^{1}\left(k, M_{i}\right)$. If we write $\alpha=\sum \alpha_{i}$ with $\alpha_{i} \in \mathrm{H}^{1}\left(k, M_{i}\right)$, then $\alpha$ is nondegenerate if and only if each $\alpha_{i} \neq 0$. When $M=N^{\oplus r}$ for a simple $G$-module $N$, the ring $R$ is the algebra of matrices of size $r$ with entries in the field $\operatorname{End}_{G}(N)$. In this case $\alpha=\sum \alpha_{i}$ is nondegenerate if and only if $\alpha_{1}, \ldots, \alpha_{r}$ are linearly independent in the $\operatorname{End}_{G}(N)$-vector space $\mathrm{H}^{1}(k, N)$.

In the following proposition we consider $M$ as a $G_{\alpha}$-module via the surjective homomorphism $\varphi: G_{\alpha} \rightarrow G$.

Proposition 3.6. With the above notation assume that $M$ is a semisimple $\Gamma_{k}$-module such that $\mathrm{H}^{1}(G, M)=0$. Let $\alpha \in \mathrm{H}^{1}(k, M)$ be a class represented by a 1-cocycle $c$. The following conditions are equivalent:

(i) the map $(c, \varphi)$ is an isomorphism of groups $G_{\alpha} \stackrel{\sim}{\longrightarrow} M \rtimes G$;

(ii) the map $\tilde{\alpha}: W_{\alpha} \stackrel{\sim}{\longrightarrow} M$ is an isomorphism of $G$-modules;

(iii) $\mathrm{H}^{1}\left(G_{\alpha}, M\right)$ is a free $R$-module generated by $\alpha$;

(iv) $\alpha$ is nondegenerate in $\mathrm{H}^{1}(k, M)$.

Proof. (i) $\Rightarrow$ (ii) Since $(c, \varphi)$ is an isomorphism, for each $m \in M$ there exists a $\gamma \in G_{\alpha}$ such that $(c, \varphi)(\gamma)=(m, 1)$. Then $\gamma$ goes to $1 \in G$ and hence $\gamma \in W_{\alpha}$ and $c(\gamma)=\tilde{\alpha}(\gamma)=m$. It follows that the map $\tilde{\alpha}: W_{\alpha} \longrightarrow M$ is surjective. Since it is also injective by construction, we conclude that it is an isomorphism of $G$-modules. (ii) $\Rightarrow$ (iii) Assume that $\tilde{\alpha}: W_{\alpha} \stackrel{\sim}{\rightarrow} M$ is an isomorphism of $G$-modules. Then $\operatorname{Hom}_{G}\left(W_{\alpha}, M\right)$ is a free $R$-module with generator $\tilde{\alpha}$. The inflation-restriction exact sequence

$$
0 \rightarrow \mathrm{H}^{1}(G, M) \rightarrow \mathrm{H}^{1}\left(G_{\alpha}, M\right) \rightarrow \mathrm{H}^{1}\left(W_{\alpha}, M\right)^{G}=\operatorname{Hom}_{G}\left(W_{\alpha}, M\right)
$$

is an exact sequence of $R$-modules. We note that $Z_{\alpha}\left(K_{\alpha}\right) \neq \varnothing$, so $\alpha \in \mathrm{H}^{1}(k, M)$ belongs to the kernel $\mathrm{H}^{1}\left(G_{\alpha}, M\right)$ of the restriction map to $\mathrm{H}^{1}\left(K_{\alpha}, M\right)$. By assumption $\mathrm{H}^{1}(G, M)=0$, hence the map $\mathrm{H}^{1}\left(G_{\alpha}, M\right) \rightarrow \operatorname{Hom}_{G}\left(W_{\alpha}, M\right)$ is injective. This map of $R$-modules sends $\alpha$ to the generator $\tilde{\alpha}$ of the $R$-module $\operatorname{Hom}_{G}\left(W_{\alpha}, M\right)=R \tilde{\alpha}$, so it is surjective, hence an isomorphism. We obtain that $\mathrm{H}^{1}\left(G_{\alpha}, M\right)$ is a free $R$-module generated by $\alpha$.

(iii) $\Rightarrow$ (iv) Assume that $\mathrm{H}^{1}\left(G_{\alpha}, M\right)$ is a free $R$-module with generator $\alpha$. By the inflation-restriction exact sequence for $\Gamma_{K_{\alpha}} \subseteq \Gamma_{k}$, the map $\mathrm{H}^{1}\left(G_{\alpha}, M\right) \rightarrow \mathrm{H}^{1}(k, M)$ is injective, and so (iv) holds.

(iv) $\Rightarrow$ (i) Suppose that $\alpha$ is nondegenerate in $\mathrm{H}^{1}(k, M)$ and assume for contradiction that the map $(c, \varphi)$ is not an isomorphism. Since $(c, \varphi)$ is injective by construction, 
we conclude that it is not surjective. The intersection of the image of $(c, \varphi)$ with $M$ is then a proper $G$-submodule $\tilde{\alpha}\left(W_{\alpha}\right) \subsetneq M$. Since $M$ is semisimple, $\tilde{\alpha}\left(W_{\alpha}\right)$ is a direct summand of $M$; see Remark 3.1. It follows that there exists a nonzero element $r \in R$ such that $r \tilde{\alpha}\left(W_{\alpha}\right)=0$, so that $r \tilde{\alpha}=0$ in $\operatorname{Hom}_{G}\left(W_{\alpha}, M\right)$. From (5) we see that $r \alpha=0$ in $\mathrm{H}^{1}\left(G_{\alpha}, M\right)$. But this is a contradiction because the map $\mathrm{H}^{1}\left(G_{\alpha}, M\right) \rightarrow \mathrm{H}^{1}(k, M)$ is injective and $\alpha$ is nondegenerate in $\mathrm{H}^{1}(k, M)$.

We record an amusing corollary of this proposition.

Corollary 3.7. Under the assumptions of Proposition 3.6, let $\alpha, \beta \in \mathrm{H}^{1}(k, M)$ be nondegenerate. Then the associated torsors $Z_{\alpha}, Z_{\beta}$ for $\mathcal{G}_{M}$ are integral $k$-schemes. Furthermore, the following conditions are equivalent:

(i) there exists an $r \in R^{*}$ such that $r \alpha=\beta$;

(ii) $R \alpha=R \beta \subset \mathrm{H}^{1}(k, M)$;

(iii) $Z_{\alpha}$ and $Z_{\beta}$ are isomorphic as abstract $k$-schemes.

Proof. Let $c$ be a cocycle representing $\alpha$. By Proposition 3.6 the group $G_{\alpha}$ acts on $Z_{\alpha}(\bar{k}) \simeq M$ via the isomorphism $(c, \varphi): G_{\alpha} \stackrel{\sim}{\longrightarrow} M \rtimes G$. Hence $G_{\alpha}$ acts transitively on $Z_{\alpha}(\bar{k})$, because already the subgroup $M \subset M \rtimes G$ acts (simply) transitively on $M$. Hence $Z_{\alpha}$ is integral. The same argument proves that $Z_{\beta}$ is integral.

Let us now establish the equivalence of (i), (ii) and (iii). The implication (i) $\Rightarrow$ (ii) is clear. Conversely, if $R \alpha=R \beta$, then there exist $r, s \in R$ such that $r \alpha=\beta$ and $\alpha=s \beta$. Then $\operatorname{sr} \alpha=\alpha$ and $r s \beta=\beta$. Since $\alpha$ and $\beta$ are nondegenerate, we obtain that $r$ and $s$ are invertible in $R$, so (ii) implies (i).

We now show that (i) is equivalent to (iii). Assume (i) and take a cocycle $c: \Gamma_{k} \rightarrow M$ which represents $\alpha$. Then $r c$ represents $\beta$. We identify $Z_{\alpha}(\bar{k})$ with $\mathcal{G}_{M}(\bar{k})$ such that $\Gamma_{k}$ acts via its original action on $\mathcal{G}_{M}(\bar{k})$ followed by the translation by $c$. Then $Z_{\beta}(\bar{k})$ can be identified with $\mathcal{G}_{M}(\bar{k})$ such that $\Gamma_{k}$ acts via its original action on $\mathcal{G}_{M}(\bar{k})$ followed by the translation by $r c$. Under these identifications the map $r: \mathcal{G}_{M}(\bar{k}) \rightarrow \mathcal{G}_{M}(\bar{k})$ becomes a $\Gamma_{k}$-equivariant map $Z_{\alpha}(\bar{k}) \rightarrow Z_{\beta}(\bar{k})$. Thus $Z_{\alpha}$ and $Z_{\beta}$ are isomorphic as 0 -dimensional $k$-schemes.

Finally, assume that $Z_{\alpha}$ and $Z_{\beta}$ are isomorphic as $k$-schemes. Since $\alpha$ and $\beta$ are nondegenerate, we see from Proposition 3.6 that the maps $\tilde{\alpha}: W_{\alpha} \stackrel{\sim}{\longrightarrow} M$ and $\tilde{\beta}: W_{\beta} \stackrel{\sim}{\longrightarrow} M$ are isomorphisms of $G$-modules. The splitting fields $K_{\alpha}$ and $K_{\beta}$ coincide as subfields of $\bar{k}$, so there exists an isomorphism of $\Gamma_{k}$-modules represented by the dotted arrow in the diagram

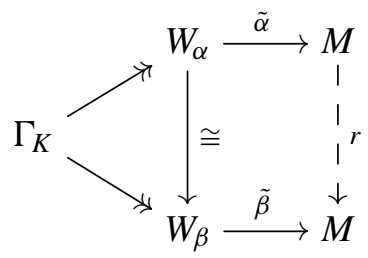


It is obtained as the action of an invertible element $r \in R^{*}$. It follows that $r \alpha$ and $\beta$ have the same image in $\mathrm{H}^{1}(K, M)$. By assumption $\mathrm{H}^{1}(G, M)=0$, hence the restriction-inflation exact sequence implies that the map $\mathrm{H}^{1}(k, M) \rightarrow \mathrm{H}^{1}(K, M)$ is injective. Thus $r \alpha=\beta$, as desired.

A continuous action of the procyclic group $\hat{\mathbb{Z}}$ on a discrete module $N$ is determined by the homomorphism $g: N \rightarrow N$ which is the action of the generator $1 \in \hat{\mathbb{Z}}$. There is a canonical isomorphism

$$
\mathrm{H}^{1}(\hat{\mathbb{Z}}, N) \cong N /(g-1)
$$

induced by sending the class of a cocycle $\xi$ to the class of $\xi(1)$ in $N /(g-1)$.

An element $\gamma \in G_{\alpha}$ determines a map $f_{\gamma}: \hat{\mathbb{Z}} \rightarrow G_{\alpha}$ which sends 1 to $\gamma$, and hence an induced map

$$
f_{\gamma}^{*}: \mathrm{H}^{1}\left(G_{\alpha}, M\right) \longrightarrow \mathrm{H}^{1}(\hat{\mathbb{Z}}, M)=M /(g-1) .
$$

Here we denote by $g$ the image of $\gamma$ in $G$ (which acts on $M$ ) under the natural surjective map $G_{\alpha} \rightarrow G$. In particular, if $c: G_{\alpha} \rightarrow M$ is a cocycle representing $\alpha \in \mathrm{H}^{1}\left(G_{\alpha}, M\right)$, then $f_{\gamma}^{*}(\alpha)$ is equal to the class of $c(\gamma)$ in $M /(g-1)$.

Corollary 3.8. In the assumptions of Proposition 3.6 let $\alpha \in \mathrm{H}^{1}(k, M)$ be nondegenerate. Take any $g \in G$ and any $x \in M /(g-1)$. Then $g$ has a lifting $\gamma \in G_{\alpha}$ such that $f_{\gamma}(\alpha)=x$.

Proof. Let $c: G_{\alpha} \rightarrow M$ be a cocycle representing $\alpha$ and let $m \in M$ be an element whose class in $M /(g-1)$ is $x$. By Proposition 3.6 the map $(c, \varphi): G_{\alpha} \stackrel{\sim}{\longrightarrow} M \rtimes G$ is an isomorphism. Hence there exists an element $\gamma \in G_{\alpha}$ such that $(c, \varphi)(\gamma)=(m, g)$. Then $\gamma$ is a lifting of $g$ and $c(\gamma)=m$ so that $f_{\gamma}(\alpha)=x$, as desired.

Corollary 3.9. Let $M$ be a semisimple $\Gamma_{k}$-module such that $G$ contains more than one element, the action of $G$ on each simple factor of $M$ is faithful and $\mathrm{H}^{1}(G, M)=0$. Let $\alpha \in \mathrm{H}^{1}(k, M)$ be nondegenerate. Then

(i) each subfield of $K_{\alpha}$ that is Galois over $k$ is either contained in $K$ or contains $K$;

(ii) each subfield of $K_{\alpha}$ that is abelian over $k$ is contained in $K$.

Proof. By Proposition 3.6 we have $G_{\alpha} \simeq M \rtimes G$. The desired result now follows directly from Lemma 3.2.

Until the end of this section we assume that $k$ is a field of characteristic different from 2. Let $A_{1}, \ldots, A_{r}$ be abelian varieties satisfying conditions (a) and (b) of Section 2 and let $A=\prod_{i=1}^{r} A_{i}$. Let $K_{i}$ be the splitting field of $A_{i}$ [2]. The compositum $K=K_{1} \cdots K_{r}$ is the field of definition of $A$ [2]. Assume that condition (e) of Section 2 holds, i.e., the fields $K_{1}, \ldots, K_{r}$ are linearly disjoint over $k$. 
Remark 3.10. Condition (a) implies that each $K_{i}$ is a nontrivial extension of $k$, so that $G_{i}$ has more than one element.

We now present two applications of the results above. In the first one we consider the semisimple $\Gamma_{k}$-module $M=A[2]=\bigoplus_{i=1}^{r} A_{i}[2]$.

Proposition 3.11. Suppose that abelian varieties $A_{1}, \ldots, A_{r}$ satisfy conditions (a) and (b), and that condition (e) holds. Let $Z_{i}$ be a nontrivial $k$-torsor for $A_{i}[2]$, for each $i=1, \ldots, r$, and let $Z=\prod_{i=1}^{r} Z_{i}$. Let $L$ be the étale $k$-algebra $k[Z]$, so that $Z \cong \operatorname{Spec}(L)$. Then $L$ is a field which contains no quadratic extension of $k$.

Proof. Let $M=A[2]$ and let $\alpha \in \mathrm{H}^{1}(k, M)$ be the class of $Z$. Write $\alpha=\sum_{i=1}^{r} \alpha_{i}$, where each $\alpha_{i} \in \mathrm{H}^{1}\left(k, A_{i}\right.$ [2]) is nonzero. By condition (a) each $A_{i}$ [2] is simple and hence $M$ is semisimple with simple factors $A_{1}[2], \ldots, A_{r}[2]$. By condition (e) the fields $K_{1}, \ldots, K_{r}$ are linearly disjoint over $k$, so that the Galois group $G=$ $\operatorname{Gal}(K / k)$ is the product $G=\prod_{i=1}^{r} G_{i}$, and the $A_{i}[2]$ are pairwise nonisomorphic $\Gamma_{k}$-modules. From Remark 3.5 we see that $\alpha$ is nondegenerate.

For each $i=1, \ldots, r$, we have $A_{i}[2]^{G_{i}}=0$ and $\mathrm{H}^{1}\left(G_{i}, A_{i}[2]\right)=0$ by conditions (a) and (b). The inflation-restriction exact sequence for $G_{i} \subset G$ then gives $\mathrm{H}^{1}\left(G, A_{i}[2]\right)=0$, and so $\mathrm{H}^{1}(G, M)=0$. Let $c: \Gamma_{k} \rightarrow M$ be a cocycle representing $\alpha$. By Proposition 3.6 the map $(c, \varphi): G_{\alpha} \stackrel{\sim}{\rightarrow} M \rtimes G$ is an isomorphism. Let $s: G \rightarrow G_{\alpha}$ be the section corresponding to the canonical section $G \rightarrow M \rtimes G$ under the isomorphism $(c, \varphi)$.

By Corollary 3.7 the scheme $Z_{\alpha}$ is integral, and hence $L=k\left[Z_{\alpha}\right]$ is a field whose Galois closure is $K_{\alpha}$ by definition. Moreover, $L \cong\left(K_{\alpha}\right)^{s(G)}$. If $L$ contains a quadratic extension of $k$, then $s(G)$ is contained in a normal subgroup $H \subset G_{\alpha}$ of index 2 . Since $s$ is a section, the induced homomorphism $H \rightarrow G$ is surjective, so its kernel is a $G$-submodule of $M$ which is a subgroup of $M$ of index 2 . But this is a contradiction since $M$ is semisimple and the simple factors $A_{i}$ [2] of $M$ have size 4 .

In the second application we consider the semisimple module $M=A_{1}[2]^{\oplus r}$.

Proposition 3.12. Suppose that abelian varieties $A_{1}, \ldots, A_{r}$ satisfy conditions (a) and (b), and that condition (e) holds. Let $M=A_{1}[2]^{\oplus r}$ be a direct sum of copies of $A_{1}[2]$ and let $\alpha \in \mathrm{H}^{1}(k, M)$ be nondegenerate. Then the fields $\left(K_{1}\right)_{\alpha}, K_{2}, \ldots, K_{r}$ are linearly disjoint.

Proof. Write $E=\left(K_{1}\right)_{\alpha} \cap K_{2} \cdots K_{r}$. In view of condition (e) it is enough to show that $E=k$. Indeed, $E$ is a Galois subfield of $\left(K_{1}\right)_{\alpha}$, so by Remark 3.10 and Corollary 3.9 we have $E \subset K_{1}$ or $K_{1} \subset E$. In the first case, $E=k$ because $E$ is contained in $K_{1} \cap K_{2} \cdots K_{r}=k$, where the equality holds by condition (e). By the same condition the second case cannot actually occur, because then $K_{1} \subset E \subset$ $K_{2} \cdots K_{r}$ which contradicts the linear disjointness of $K_{1}, \ldots, K_{r}$. 


\section{Kummer map over a local field}

Let $A$ be an abelian variety over a local field $k$ of characteristic zero. The Kummer exact sequence gives rise to a map $\delta: A(k) \rightarrow \mathrm{H}^{1}(k, A[2])$, called the Kummer map. For $x \in A(k)$, choose $\bar{x} \in A(\bar{k})$ such that $2 \bar{x}=x$. Then $\delta(x)$ is represented by the cocycle that sends $\gamma \in \Gamma_{k}$ to $\gamma_{\bar{x}}-\bar{x} \in A[2]$.

The Weil pairing is a nondegenerate pairing of $\Gamma_{k}$-modules $A[2] \times A^{t}[2] \rightarrow \mathbb{Z} / 2$. The induced pairing on cohomology followed by the local invariant of local class field theory gives a nondegenerate pairing of finite abelian groups [Milne 1986, Corollary I.2.3]

$$
\mathrm{H}^{1}(k, A[2]) \times \mathrm{H}^{1}\left(k, A^{t}[2]\right) \longrightarrow \mathrm{Br}(k)[2] \stackrel{\text { inv }}{\longrightarrow} \frac{1}{2} \mathbb{Z} / \mathbb{Z} .
$$

The local Tate duality implies that $\delta(A(k))$ and $\delta\left(A^{t}(k)\right)$ are the orthogonal complements to each other under this pairing (see, e.g., the first commutative diagram in the proof of [Milne 1986, I.3.2]).

When $A$ is principally polarised, we combine the last pairing with the principal polarisation $A \stackrel{\sim}{\longrightarrow} A^{t}$ and obtain a nondegenerate symmetric pairing

$$
\operatorname{inv}(\alpha \cup \beta): \mathrm{H}^{1}(k, A[2]) \times \mathrm{H}^{1}(k, A[2]) \longrightarrow \operatorname{Br}(k)[2] \stackrel{\text { inv }}{\longrightarrow} \frac{1}{2} \mathbb{Z} / \mathbb{Z} .
$$

It is well-known that $\delta(A(k))$ is a maximal isotropic subspace of $\mathrm{H}^{1}(k, A[2])$; see [Poonen and Rains 2012, Proposition 4.11]. Note that the pairing $\operatorname{inv}(\alpha \cup \beta)$ is also defined for $k=\mathbb{R}$ and the above statements carry over to this case; cf. [Milne 1986, Theorem I.2.13(a), Remark I.3.7].

Let us recall a well-known description of $\delta(A(k))$ when $A$ has good reduction. Let $\kappa$ be the residue field of $k$, and assume $\operatorname{char}(\kappa)=\ell \neq 2$. Then $\delta(A(k))$ is the unramified subgroup

$$
\mathrm{H}_{\mathrm{nr}}^{1}(k, A[2])=\operatorname{Ker}\left[\mathrm{H}^{1}\left(\Gamma_{k}, A[2]\right) \longrightarrow \mathrm{H}^{1}(I, A[2])\right],
$$

where $I \subset \Gamma_{k}$ is the inertia subgroup. By Néron-Ogg-Shafarevich the inertia acts trivially on $A$ [2], so that $\mathrm{H}_{\mathrm{nr}}^{1}(k, A[2])=\mathrm{H}^{1}(\kappa, A[2])$. The absolute Galois group $\operatorname{Gal}(\bar{\kappa} / \kappa)=\Gamma_{k} / I$ is isomorphic to $\hat{\mathbb{Z}}$ with the Frobenius element as a topological generator. Thus we have a canonical isomorphism

$$
\delta(A(k))=A[2] /(\text { Frob }-1) .
$$

Since $\hat{\mathbb{Z}}$ has cohomological dimension 1, the spectral sequence

$$
\mathrm{H}^{p}\left(\hat{\mathbb{Z}}, \mathrm{H}^{q}(I, A[2])\right) \Rightarrow \mathrm{H}^{p+q}(k, A[2])
$$

gives rise to the exact sequence

$$
0 \rightarrow A[2] /(\text { Frob }-1) \rightarrow \mathrm{H}^{1}(k, A[2]) \rightarrow \operatorname{Hom}(I, A[2])^{\text {Frob }} \rightarrow 0 .
$$


The maximal abelian pro-2-quotient of $I$ is isomorphic to $\mathbb{Z}_{2}$, and Frob acts on it by multiplication by $\ell$. Thus $\operatorname{Hom}(I, A[2])=A[2]$ with the natural action of Frob, so that

$$
\operatorname{Hom}(I, A[2])^{\text {Frob }}=A[2]^{\text {Frob }}=\operatorname{Ker}(\text { Frob }-1: A[2] \rightarrow A[2]) .
$$

It follows that the dimension of the $\mathbb{F}_{2}$-vector space $A[2] /($ Frob -1$)$ equals the dimension of $A[2]^{\text {Frob }}$, and therefore

$$
\operatorname{dim} \mathrm{H}^{1}(k, A[2])=2 \operatorname{dim} A[2] /(\text { Frob }-1) .
$$

Let us now return to the general case, where $A$ does not necessarily have good reduction. If $F / k$ is a quadratic extension, we write $\delta^{F}: A^{F}(k) \rightarrow \mathrm{H}^{1}(k, A[2])$ for the Kummer map of $A^{F}$. In the rest of this section we summarise some known results relating $\delta, \delta^{F}$ and the norm map $\mathrm{N}: A(F) \rightarrow A(k)$.

Lemma 4.1. We have $\delta(\mathrm{N}(A(F)))=\delta(A(k)) \cap \delta^{F}\left(A^{F}(k)\right) \subset \mathrm{H}^{1}(k, A[2])$.

Proof. Cf. [Kramer 1981, Proposition 7] or [Mazur and Rubin 2007, Proposition 5.2]. Let $\chi: \Gamma_{k} \rightarrow\{ \pm 1\}$ be the quadratic character associated to $F$. We choose $\sigma \in \Gamma_{k}$ such that $\chi(\sigma)=-1$.

Suppose that $x \in A(k)$ and $y \in A^{F}(k)$ are such that $\delta(x)=\delta^{F}(y)$. Using the embedding $A^{F}(k) \subset A(F)$ we can consider $y$ as a point in $A(F)$ such that ${ }^{\sigma} y=-y$. If $\bar{y} \in A(\bar{k})$ is such that $2 \bar{y}=y$, then $\delta^{F}(y)$ is represented by the cocycle that sends $\gamma \in \Gamma_{k}$ to

$$
\chi(\gamma)^{\gamma} \bar{y}-\bar{y}={ }^{\gamma} \bar{y}-\chi(\gamma) \bar{y} \in A[2] .
$$

Since $\delta(x)=\delta^{F}(y)$, we can choose $\bar{x} \in A(\bar{k})$ such that $2 \bar{x}=x$ and such that

$$
\chi(\gamma)^{\gamma} \bar{y}-\bar{y}={ }^{\gamma} \bar{x}-\bar{x}
$$

We deduce that $\gamma(\bar{x}-\bar{y})=\bar{x}-\chi(\gamma) \bar{y}$ for every $\gamma \in \Gamma_{k}$. It follows that $\bar{x}-\bar{y} \in A(F)$ and ${ }^{\sigma}(\bar{x}-\bar{y})=\bar{x}+\bar{y}$. Therefore, $x=2 \bar{x}=\mathrm{N}(\bar{x}-\bar{y})$ is a norm from $A(F)$.

Conversely, suppose that $x=\mathrm{N}(z)=z+{ }^{\sigma} z$ for some $z \in A(F)$. Let $y={ }^{\sigma} z-z$. Then $y \in A^{F}(k)$ and we claim that $\delta(x)=\delta^{F}(y)$. Choose $\bar{x} \in A(\bar{k})$ such that $2 \bar{x}=x$ and set $\bar{y}=\bar{x}-z$. Then $2 \bar{y}=x-2 z=y$ and we have $\bar{x}-\bar{y}=z$ and $\bar{x}+\bar{y}={ }^{\sigma} z$. It follows that for each $\gamma \in \Gamma_{k}$ we have $\gamma(\bar{x}-\bar{y})=\bar{x}-\chi(\gamma) \bar{y}$, and hence

$$
\gamma_{\bar{x}}-\bar{x}=\gamma_{\bar{y}}-\chi(\gamma) \bar{y} \text {. }
$$

This implies $\delta(x)=\delta^{F}(y)$, as desired.

Lemma 4.2. Let $A$ be a principally polarised abelian variety over $k$ with bad reduction such that the number of geometric connected components of the Néron model of $A$ is odd. If $F$ is an unramified quadratic extension of $k$, then $\delta(A(k))=\delta^{F}\left(A^{F}(k)\right)$. 
Proof. Since $A$ is principally polarised, it is isomorphic to its dual abelian variety. It follows from [Mazur 1972, Propositions 4.2 and 4.3] that the norm map $\mathrm{N}: A(F) \rightarrow A(k)$ is surjective. By Lemma 4.1 we see that $\delta(A(k)) \subset \delta^{F}\left(A^{F}(k)\right)$. Since $F$ is unramified, the quadratic twist $A^{F}$ also satisfies the assumptions of the lemma, and the same argument applied to $A^{F}$ gives the opposite inclusion.

Lemma 4.3. Assume that the residue characteristic of $k$ is not 2. If $A$ is an abelian variety over $k$ with good reduction and $F$ is a ramified quadratic extension of $k$, then $\delta(A(k)) \cap \delta^{F}\left(A^{F}(k)\right)=0$.

Proof. In this case we have $\mathrm{N}(A(F))=2 A(k)$. If $\operatorname{dim}(A)=1$ this is proved in [Mazur and Rubin 2007, Lemma 5.5(ii)], and the same proof works in the general case. It remains to apply Lemma 4.1 .

\section{Selmer group and Cassels-Tate pairing}

Let $A$ be an abelian variety over a field $k$ of characteristic zero. Let $\operatorname{NS}(\bar{A})$ be the Néron-Severi group of $\bar{A}$. The dual abelian variety $A^{t}$ represents the functor $\mathrm{Pic}_{A}^{0}$. In particular, we have an exact sequence of $\Gamma_{k}$-modules

$$
0 \longrightarrow A^{t}(\bar{k}) \longrightarrow \operatorname{Pic}(\bar{A}) \longrightarrow \mathrm{NS}(\bar{A}) \longrightarrow 0 .
$$

The antipodal involution $\iota_{A}=[-1]: A \rightarrow A$ induces an action of $\mathbb{Z} / 2$ on $\operatorname{Pic}(\bar{A})$ which turns $(8)$ into an exact sequence of $\mathbb{Z} / 2$-modules. The induced action on $\operatorname{NS}(\bar{A})$ is trivial; see [Skorobogatov and Zarhin 2012, p. 119]. The involution $\iota_{A}$ induces the involution $\iota_{A^{t}}$ on $A^{t}$. Since $A^{t}(\bar{k})$ is divisible, we obtain $\mathrm{H}^{1}\left(\mathbb{Z} / 2, A^{t}(\bar{k})\right)=0$. Thus the long exact sequence of cohomology gives an exact sequence

$$
0 \longrightarrow A^{t}[2] \longrightarrow \operatorname{Pic}(\bar{A})^{[-1]^{*}} \longrightarrow \mathrm{NS}(\bar{A}) \longrightarrow 0
$$

cf. [Poonen and Rains 2011, Section 3.2]. It is well-known that NS $(\bar{A})$ is canonically isomorphic to the group $\operatorname{Hom}\left(\bar{A}, \bar{A}^{t}\right)^{\text {sym }}$ of self-dual homomorphisms of abelian varieties $\bar{A} \rightarrow \bar{A}^{t}$; see, e.g., [Polishchuk 2003, Theorem 13.7]. Hence $\operatorname{NS}(\bar{A})^{\Gamma_{k}}$ is canonically isomorphic to the group $\operatorname{Hom}\left(A, A^{t}\right)^{\mathrm{sym}}$ of self-dual $k$-homomorphisms of abelian varieties $A \rightarrow A^{t}$; cf. [Poonen and Rains 2011, Remark 3.3]. A polarisation on $A$ is an element $\lambda \in \operatorname{NS}(\bar{A})^{\Gamma_{k}}$ that comes from an ample line bundle on $\bar{A}$. The polarisation is called principal if the associated morphism $\varphi_{\lambda}: A \rightarrow A^{t}$ is an isomorphism. Following [Poonen and Rains 2011] we shall write $c_{\lambda}$ for the image of $\lambda$ under the differential $\mathrm{NS}(\bar{A})^{\Gamma_{k}} \rightarrow \mathrm{H}^{1}\left(k, A^{t}[2]\right)$ attached to (9). In particular, $c_{\lambda}$ vanishes if and only if $\lambda$ lifts to an element of $\left(\operatorname{Pic}(\bar{A})^{[-1]^{*}}\right)^{\Gamma_{k}}=\operatorname{Pic}(A)^{[-1]^{*}}$. For example, if $A$ is the Jacobian of a smooth projective curve $C$ and $\lambda$ is the canonical principal polarisation of $A$, then $c_{\lambda}$ is the image of the class of the theta characteristics torsor of $C$ under the isomorphism $\varphi_{\lambda *}: \mathrm{H}^{1}(k, A[2]) \stackrel{\sim}{\longrightarrow} \mathrm{H}^{1}\left(k, A^{t}[2]\right)$; see 
[Poonen and Rains 2011, Theorem 3.9]. In this case, $c_{\lambda}=0$ when $C$ has a rational Weierstrass point.

Lemma 5.1. Let $A$ be an abelian variety over a field $k$ of characteristic 0 with polarisation $\lambda$. Let $K=k(A[2])$. Then $c_{\lambda}$ belongs to the kernel of the restriction map $\mathrm{H}^{1}\left(k, A^{t}[2]\right) \rightarrow \mathrm{H}^{1}\left(K, A^{t}[2]\right)$.

Proof. This is a particular case of [Poonen and Rains 2011, Lemma 3.6(a)].

Now let $k$ be a number field. For a place $v$ of $k$, let

$$
\operatorname{loc}_{v}: \mathrm{H}^{1}(k, A[2]) \longrightarrow \mathrm{H}^{1}\left(k_{v}, A[2]\right)
$$

be the natural restriction map. The 2-Selmer group $\operatorname{Sel}_{2}(A) \subset \mathrm{H}^{1}(k, A[2])$ is defined as the set of elements $x$ such that $\operatorname{loc}_{v}(x) \in \delta\left(A\left(k_{v}\right)\right)$ for all places $v$ of $k$. If $v$ is a place of good reduction, then (6) allows us to write the restriction map at $v$ as

$$
\operatorname{loc}_{v}: \operatorname{Sel}_{2}(A) \longrightarrow A[2] /\left(\operatorname{Frob}_{v}-1\right) .
$$

For every quadratic extension $F / k$, we have $A^{F}[2]=A[2]$ and hence we may consider the 2-Selmer groups $\operatorname{Sel}_{2}\left(A^{F}\right)$ of all quadratic twists $A^{F}$ as subgroups of $\mathrm{H}^{1}(k, A[2])$. We have the well-known exact sequence

$$
0 \longrightarrow A(k) / 2 \longrightarrow \operatorname{Sel}_{2}(A) \longrightarrow \amalg(A)[2] \longrightarrow 0 .
$$

The Cassels-Tate pairing is a bilinear pairing

$$
\langle,\rangle: \amalg(A) \times \amalg\left(A^{t}\right) \longrightarrow \mathbb{Q} / \mathbb{Z} .
$$

If $\amalg(A)$ is finite, then $\amalg\left(A^{t}\right)$ is finite too and the Cassels-Tate pairing is nondegenerate; see [Milne 1986, Theorem I.6.26]. A polarisation $\lambda$ on $A$ induces a homomorphism $\varphi_{\lambda_{*}}: \amalg(A) \rightarrow \amalg\left(A^{t}\right)$.

Proposition 5.2. Let $A$ be an abelian variety over a number field $k$ with a principal polarisation $\lambda$. Then condition (b) of Section 2 implies that the Cassels-Tate pairing $\left\langle x, \varphi_{\lambda_{*}} y\right\rangle$ on $\amalg(A)\{2\}$ is alternating. In particular, if the 2-primary subgroup $\amalg(A)\{2\}$ is finite, then the cardinality of $\amalg(A)[2]$ is a square.

Proof. By [Poonen and Stoll 1999, Corollary 2] we know that $c_{\lambda} \in \operatorname{Sel}_{2}\left(A^{t}\right)$. If $c_{\lambda}^{\prime}$ is the image of $c_{\lambda}$ in $\amalg\left(A^{t}\right)$ [2], then [Poonen and Stoll 1999, Theorem 5] says that $\left\langle x, \varphi_{\lambda *} x+c_{\lambda}^{\prime}\right\rangle=0$ for any $x \in \amalg(A)$. Thus it is enough to prove that $c_{\lambda}=0$. Lemma 5.1 implies that $c_{\lambda}$ belongs to the image of the inflation map $\mathrm{H}^{1}\left(G, A^{t}[2]\right) \rightarrow$ $\mathrm{H}^{1}\left(k, A^{t}[2]\right)$, where $G=\mathrm{Gal}(k(A[2]) / k)$ is the image of $\Gamma_{k} \rightarrow \mathrm{GL}(A[2])$. Since $\lambda$ is a principal polarisation, $\varphi_{\lambda}$ induces an isomorphism of $\Gamma_{k}$-modules $A$ [2] $\stackrel{\sim}{\longrightarrow} A^{t}$ [2]. Now condition (b) implies $\mathrm{H}^{1}\left(G, A^{t}[2]\right)=\mathrm{H}^{1}(G, A[2])=0$, hence $c_{\lambda}=0$. 


\section{Kummer varieties}

Let $A$ be an abelian variety over a field $k$ of characteristic different from 2 . Let $Z$ be a $k$-torsor for the group $k$-scheme $A$ [2]. Recall that the 2-covering $f: Y \rightarrow A$ associated to $Z$ is a $k$-torsor for $A$ defined as the quotient of $A \times{ }_{k} Z$ by the diagonal action of $A$ [2]. In other words, $Y$ is the twisted form of $A$ by $Z$ with respect to the action of $A[2]$ by translations. The morphism $f$ is induced by the first projection, and we have $Z=f^{-1}(0)$. Let $L$ be the étale $k$-algebra $k[Z]$, so that $Z \cong \operatorname{Spec}(L)$.

Let $\tilde{Y}$ be the blowing-up of $Z$ in $Y$. The antipodal involution $\iota_{A}: A \rightarrow A$ induces the map $\left(\iota_{A}\right.$, Id) $: A \times_{k} Z \rightarrow A \times_{k} Z$ which commutes with the action of $A$ [2] and hence induces an involution $\iota_{Y}: Y \rightarrow Y$. As $\iota_{Y}$ fixes $Z=f^{-1}(0) \subseteq Y$ it extends to an involution $\iota_{\tilde{Y}}: \tilde{Y} \rightarrow \tilde{Y}$ whose fixed-point set is precisely the exceptional divisor. It is easy to see that the quotient $X=\operatorname{Kum}(Y)=\tilde{Y} / \iota_{\tilde{Y}}$ is smooth. We call $X$ the Kummer variety attached to $A$ and $Z$. We note that the branch locus of $\tilde{Y} \rightarrow X$ is $Z \times{ }_{k} \mathbb{P}_{k}^{d-1}$, where $d=\operatorname{dim}(A)$.

Let $F$ be an extension of $k$ of degree at most 2. Recall that $A^{F}$ denotes the quadratic twist of $A$ by $F$, that is, the abelian variety over $k$ obtained by twisting $A$ by the quadratic character of $F$ with respect to the action of $\mu_{2}$ via the antipodal involution $\iota_{A}$. Similarly, $Y^{F}$ denotes the quadratic twist of $Y$ with respect to the involution $\iota_{Y}$; see Section 1 . Since $\iota_{A}$ commutes with translations by the elements of $A$ [2], the quadratic twist $Y^{F}$ of $Y$ is a $k$-torsor for $A^{F}$. We have a natural embedding $i_{F}: Z \rightarrow Y^{F}$. Then $\tilde{Y}^{F}$, defined as the blowing-up of $i_{F}(Z)$ in $Y^{F}$, is the quadratic twist of $\tilde{Y}$ by the quadratic character of $F$ with respect to the action of $\mu_{2}$ on $\tilde{Y}$ via $\iota_{\tilde{Y}}$. We can also consider $\tilde{Y}^{F}$ as a quadratic twist of the 2-covering $\tilde{Y} \rightarrow X$, and consequently consider every $\tilde{Y}^{F}$ as a (ramified) 2-covering of $X$. It is clear that $Y^{F}$, and hence $X$, has a $K$-point for any extension $K / k$ such that $\alpha$ is in the kernel of the natural map $\mathrm{H}^{1}(k, A[2]) \rightarrow \mathrm{H}^{1}\left(K, A^{F}\right)$.

We now recall a construction from [Skorobogatov and Swinnerton-Dyer 2005, $\S 5]$. Let $\mathcal{Y}$ be the quotient of $\tilde{Y} \times \mathbb{G}_{m, k}$ by the action of $\mu_{2}$ in which the generator $-1 \in \mu_{2}$ acts as the multiplication by -1 on $\mathbb{G}_{m}$ and by $\iota_{\tilde{Y}}$ on $\tilde{Y}$. The fibre of $\mathcal{Y}$ over $a \in \mathbb{G}_{m, k}(k)$ can be naturally identified with the quadratic twist $\tilde{Y}^{F}$ where $F=k(\sqrt{a})$. As in [Skorobogatov and Swinnerton-Dyer 2005, §5] one may consider a smooth compactification $\mathcal{Y} \subset \mathcal{X}$ that fits into the commutative diagram

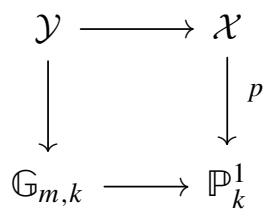

Proposition 6.1. Let $A=\prod_{i=1}^{r} A_{i}$ be a product of abelian varieties over $k$ satisfying conditions (a) and (b) of Section 2 such that condition (e) holds. Assume in addition 
that the class $\alpha \in \mathrm{H}^{1}(k, A[2])$ of $Z$ is nondegenerate (see Definition 3.4). Then the vertical Brauer group of $\mathcal{X}$ over $\mathbb{P}_{k}^{1}$ is the image of $\operatorname{Br}(k)$ in $\operatorname{Br}(\mathcal{X})$.

Proof. Let $t$ be a coordinate on $\mathbb{P}^{1}$ invertible on $\mathbb{G}_{m, k} \subset \mathbb{P}_{k}^{1}$. According to [Skorobogatov and Swinnerton-Dyer 2005, Theorem 3] the vertical Brauer group of $\mathcal{X}$ is generated by the image of $\operatorname{Br}(k)$ and the pullbacks of the classes $(t, c) \in \operatorname{Br}\left(k\left(\mathbb{P}_{k}^{1}\right)\right)$, where $c \in k^{*}$ becomes a square in $L=k[Z]$. By Proposition 3.11 the element $c$ is already a square in $k$, hence the result.

Proposition 6.2. Let $k$ be a number field. Let $A=\prod_{i=1}^{r} A_{i}$ be a product of abelian varieties over $k$ satisfying conditions (a) and (b) of Section 2, and such that conditions (e) and (f) hold. Let $Z$ be a k-torsor for $A[2]$ whose class $\alpha \in \mathrm{H}^{1}(k, A[2])$ is unramified at the places $w_{1}, \ldots, w_{r}$ and nondegenerate. Let $Y$ be the attached 2-covering of $A$ and let $X=\operatorname{Kum}(Y)$. If $X$ is everywhere locally soluble, then there exists an extension $F$ of $k$ of degree at most 2 such that $Y^{F}$ is everywhere locally soluble and $F$ is split at $w_{1}, \ldots, w_{r}$.

Proof. This is proved in [Skorobogatov and Swinnerton-Dyer 2005, Lemma 6], but we give a detailed proof for the convenience of the reader. Let $w$ be one of the places $w_{1}, \ldots, w_{r}$. By assumption $\alpha \in \mathrm{H}^{1}(k, A[2])$ goes to zero under the composed map

$$
\mathrm{H}^{1}(k, A[2]) \longrightarrow \mathrm{H}^{1}\left(k_{w}, A[2]\right) \longrightarrow \mathrm{H}^{1}\left(k_{w}^{\mathrm{nr}}, A[2]\right) .
$$

Hence the class $[Y] \in \mathrm{H}^{1}(k, A)[2]$ goes to zero under the composed map

$$
\mathrm{H}^{1}(k, A) \longrightarrow \mathrm{H}^{1}\left(k_{w}, A\right) \longrightarrow \mathrm{H}^{1}\left(k_{w}^{\mathrm{nr}}, A\right) .
$$

The second arrow in (11) is the restriction map $\mathrm{H}^{1}\left(\Gamma_{k_{w}}, A\right) \rightarrow \mathrm{H}^{1}\left(I_{w}, A\right)$, where $\Gamma_{k_{w}}=\operatorname{Gal}\left(\overline{k_{w}} / k_{w}\right)$ and $I_{w} \subset \Gamma_{k_{w}}$ is the inertia subgroup. By the inflation-restriction sequence we see that the class $\left[Y \times_{k} k_{w}\right] \in \mathrm{H}^{1}\left(\Gamma_{k_{w}}, A\right)$ belongs to the subgroup $\mathrm{H}^{1}\left(\Gamma_{k_{w}} / I_{w}, A\left(k_{w}^{\mathrm{nr}}\right)\right)$. Let $\mathcal{A} \rightarrow \operatorname{Spec}\left(\mathcal{O}_{w}\right)$ be the Néron model of $A \times_{k} k_{w}$. By [Milne 1986, Proposition I.3.8] we have an isomorphism

$$
\mathrm{H}^{1}\left(\Gamma_{k_{w}} / I_{w}, A\left(k_{w}^{\mathrm{nr}}\right)\right)=\mathrm{H}^{1}\left(\Gamma_{k_{w}} / I_{w}, \pi_{0}\left(\mathcal{A} \times \mathcal{O}_{w} \mathbb{F}_{w}\right)\right),
$$

where $\pi_{0}\left(\mathcal{A} \times \times_{\mathcal{O}_{w}} \mathbb{F}_{w}\right)$ is the group of connected components of the special fibre of $\mathcal{A} \rightarrow \operatorname{Spec}\left(\mathcal{O}_{w}\right)$. Since $2[Y]=0$, condition (f) implies that $\left[Y \times_{k} k_{w}\right]=0$, hence $Y$ has a $k_{w}$-point $P_{w} \in Y\left(k_{w}\right)$. We view $P_{w}$ as a point $\left(P_{w}, 1\right) \in \mathcal{Y}$ above $1 \in \mathbb{G}_{m, k}(k) \subset \mathbb{P}_{k}^{1}$.

For each place $v$ of $k$ and for each point $Q_{v} \in X\left(k_{v}\right)$, there exists an extension $F_{v} / k_{v}$ of degree at most 2 such that $Q_{v}$ lifts to $\tilde{Y}^{F_{v}}\left(k_{v}\right)$. Since $X$ is everywhere locally soluble, we can use this observation to extend the collection of local points $\left(P_{w}, 1\right), w \in\left\{w_{1}, \ldots, w_{r}\right\}$, to an adelic point $\left(P_{v}\right) \in \mathcal{Y}\left(\mathbb{A}_{k}\right) \subseteq \mathcal{X}\left(\mathbb{A}_{k}\right)$. The fibration $\mathcal{X} \rightarrow \mathbb{P}_{k}^{1}$ has only two bad fibres at 0 and $\infty$ (both of which are geometrically split). 
By Proposition 6.1 the vertical Brauer group of $\mathcal{X}$ over $\mathbb{P}_{k}^{1}$ is generated by the image of $\operatorname{Br}(k)$; therefore the desired result can now be obtained by applying the fibration method. More precisely, one proceeds as in the proof of [Colliot-Thélène and Skorobogatov 2000, Theorem A]. (As a more recent reference one can apply [Harpaz and Wittenberg 2016, Theorem 9.17] with $B=0$ and $U=\mathbb{G}_{m, k}$, which is justified in the light of [Harpaz and Wittenberg 2016, Theorem 9.11].) We obtain that there exists an adelic point $\left(P_{v}^{\prime}\right) \in \mathcal{X}\left(\mathbb{A}_{k}\right)$ arbitrarily close to $\left(P_{v}\right)$ such that the image of $\left(P_{v}^{\prime}\right)$ in $\mathbb{P}_{k}^{1}\left(\mathbb{A}_{k}\right)$ is a $k$-point. Let us call it $a$. By the construction of $\left(P_{v}\right)$ we can assume that $a \in \mathbb{G}_{m, k}(k)$ and that $a$ is arbitrarily close to 1 in the $w$-adic topology for $w \in\left\{w_{1}, \ldots, w_{r}\right\}$. The quadratic extension $F=k(\sqrt{a})$ now satisfies the desired properties.

\section{Proof of Theorem 2.3}

Suppose that our Kummer variety is $X=\operatorname{Kum}(Y)$, where $Y$ is the $k$-torsor for $A$ defined by a class $\alpha \in \mathrm{H}^{1}(k, A[2])$. To prove the existence of a $k$-point on $X$, it is enough to find a quadratic (or trivial) extension $F$ of $k$ such that $\alpha$ goes to 0 in $\mathrm{H}^{1}\left(k, A^{F}\right)$. We write $\alpha=\sum_{i=1}^{r} \alpha_{i}$, where $\alpha_{i} \in \mathrm{H}^{1}\left(k, A_{i}[2]\right)$ is nonzero for each $i=1, \ldots, r$. Let $K_{i}=K\left(A_{i}[2]\right)$. For each $i=1, \ldots, r$, we fix $g_{i}, h_{i} \in \operatorname{Gal}\left(K_{i} / k\right)$ satisfying conditions (c) and (d), respectively, for $A_{i}$.

By Proposition 6.2 there is a quadratic extension $F$ of $k$ split at $w_{1}, \ldots, w_{r}$ such that $\alpha \in \operatorname{Sel}_{2}\left(A^{F}\right)$. Replacing $A$ with $A^{F}$, we can assume without loss of generality that $\alpha \in \operatorname{Sel}_{2}(A)$. By doing so we preserve conditions (a), (b), (c), (d), (e) and $(\mathrm{g})$, which are not affected by quadratic twisting. The extension $F / k$ is split at $w_{1}, \ldots, w_{r}$, so replacing $A$ by $A^{F}$ also preserves condition (f) for each $A_{i}$.

Let $S_{0}$ be the set of places of $k$ that contains all the Archimedean places and the places above 2 .

Lemma 7.1. Let $S$ be the set of places of $k$ which is the union of $S_{0}$ and all the places of bad reduction of $A$ excluding $w_{1}, \ldots, w_{r}$. For each $i=1, \ldots, r$, let $\alpha_{i} \in \operatorname{Sel}_{2}\left(A_{i}\right)$ be nonzero. Let $\beta \in \operatorname{Sel}_{2}\left(A_{1}\right)$ be a nonzero class such that $\beta \neq \alpha_{1}$. Then there exists a $q \in k^{*}$ such that $\mathfrak{q}=(q)$ is a prime ideal of $k$ with the following properties:

(1) all the places in $S$ (including the Archimedean places) are split in $F=k(\sqrt{q})$; in particular, $\mathfrak{q} \notin S$;

(2) A has good reduction at $\mathfrak{q}$;

(3) Frob $_{\mathfrak{q}}$ acts on $A_{1}[2]$ as $g_{1}$;

(4) Frob $_{\mathfrak{q}}$ acts on $A_{i}[2]$ as $h_{i}$, for each $i \neq 1$;

(5) $\operatorname{loc}_{\mathfrak{q}}\left(\alpha_{1}\right)=0$, but $\operatorname{loc}_{\mathfrak{q}}(\beta) \neq 0$. 
Proof. We adapt the arguments from the proof of Proposition 5.1 in [Mazur and Rubin 2010].

Let $M=A_{1}[2]^{\oplus 2}$ be the direct sum of two copies of $A_{1}[2]$. Let

$$
\alpha=\alpha_{1}+\beta \in \mathrm{H}^{1}\left(k, A_{1}[2]\right) \oplus \mathrm{H}^{1}\left(k, A_{1}[2]\right)=\mathrm{H}^{1}(k, M) .
$$

The splitting field of $M$ is $K_{1}$ and the Galois action on $M$ factors through $G_{1}=$ $\operatorname{Gal}\left(K_{1} / k\right)$. Let $\left(K_{1}\right)_{\alpha}$ and $\left(G_{1}\right)_{\alpha}$ be as in Definition 3.3. By Corollary 3.8 we can find a lift $\gamma \in\left(G_{1}\right)_{\alpha}$ of $g \in G_{1}$ such that the associated map

$$
f_{\gamma}: \mathrm{H}^{1}(k, M) \longrightarrow M /(g-1)=\left(A_{1}[2] /(g-1)\right) \oplus\left(A_{1}[2] /(g-1)\right)=\mathbb{Z} / 2 \oplus \mathbb{Z} / 2
$$

sends $\alpha$ to the class $(0,1) \in \mathbb{Z} / 2 \oplus \mathbb{Z} / 2$. That is, $f_{\gamma}\left(\alpha_{1}, 0\right)=0$, whereas $f_{\gamma}(0, \beta) \neq 0$.

The fields $\left(K_{1}\right)_{\alpha}, K_{2}, \ldots, K_{r}$ are Galois extensions of $k$ that are linearly disjoint by condition (e) and Proposition 3.12. Let $\mathcal{K}$ be the compositum of $\left(K_{1}\right)_{\alpha}, K_{2}, \ldots, K_{r}$. This is a Galois extension of $k$ with the Galois group $\operatorname{Gal}(\mathcal{K} / k)=\left(G_{1}\right)_{\alpha} \times \prod_{i=2}^{r} G_{i}$.

Let the modulus $\mathfrak{m}$ be the formal product of the real places of $k, 8$ and all the odd primes in $S$. Let $k_{\mathfrak{m}}$ be the ray class field associated to the modulus $\mathfrak{m}$. This is an abelian extension of $k$ which is unramified away from $\mathfrak{m}$. We claim that $k_{\mathfrak{m}}$ and $\mathcal{K}$ are linearly disjoint over $k$. Indeed, $k^{\prime}=k_{\mathfrak{m}} \cap \mathcal{K}$ is a subfield of $\mathcal{K}$ that is abelian over $k$ and unramified at $w_{1}, \ldots, w_{r}$. We note that $\operatorname{Gal}(\mathcal{K} / k)^{\mathrm{ab}}=\left(G_{1}\right)_{\alpha}^{\mathrm{ab}} \times \prod_{i=2}^{r} G_{i}^{\mathrm{ab}}$. By Corollary 3.9 (applicable in the light of Remark 3.10) we have $\left(G_{1}\right)_{\alpha}^{\mathrm{ab}}=\left(G_{1}\right)^{\mathrm{ab}}$. Therefore, $\operatorname{Gal}(\mathcal{K} / k)^{\mathrm{ab}}=\prod_{i=1}^{r} G_{i}^{\mathrm{ab}}$, so that $k^{\prime}$ is contained in the compositum $L=k_{1}^{\mathrm{ab}} \cdots k_{r}^{\mathrm{ab}}$ of linearly disjoint abelian extensions $k_{1}^{\mathrm{ab}}, \ldots, k_{r}^{\mathrm{ab}}$, where, as in Section $2, k_{i}^{\mathrm{ab}}$ denotes the maximal abelian subextension of $K_{i} / k$.

Write $M=k_{1}^{\mathrm{ab}} \cdots k_{r-1}^{\mathrm{ab}}$. The extension $k_{r}^{\mathrm{ab}} / k$ is totally ramified at $w_{r}$ by condition $(\mathrm{g})$, whereas $k^{\prime} / k$ and $M / k$ are unramified at $w_{r}$ (the latter by the criterion of NéronOgg-Shafarevich). Hence $L / M$ is totally ramified at each prime $v$ of $M$ over $w_{r}$. Since $M \subset k^{\prime} M \subset L$, where $k^{\prime} M / M$ is unramified over $v$, we must have $k^{\prime} \subset M$. Continuing by induction, we prove that $k^{\prime}=k$, as required.

It follows that $k_{\mathfrak{m}} \mathcal{K}$ is a Galois extension of $k$ with the Galois group

$$
\operatorname{Gal}\left(k_{\mathfrak{m}} \mathcal{K} / k\right)=\operatorname{Gal}\left(k_{\mathfrak{m}} / k\right) \times\left(G_{1}\right)_{\alpha} \times \prod_{i=2}^{r} G_{i} .
$$

By Chebotarev's density theorem we can find a place $\mathfrak{q}$ of $k$ such that the corresponding Frobenius element in $\operatorname{Gal}\left(k_{\mathfrak{m}} \mathcal{K} / k\right)$ is the conjugacy class of $\left(1, \gamma, h_{2}, \ldots, h_{r}\right)$. Then $\mathfrak{q}$ is a principal prime ideal with a totally positive generator $q \equiv 1 \bmod 8$, hence $q$ is a square in each completion of $k$ at a prime over 2 . We also have $q \equiv 1 \bmod \mathfrak{p}$ for any odd $\mathfrak{p} \in S$. Thus all the places of $S$ including the Archimedean places are split in $F=k(\sqrt{q})$. All other conditions are satisfied by construction. 
Proposition 7.2. For any $\beta \in \operatorname{Sel}_{2}\left(A_{1}\right), \beta \neq 0, \beta \neq \alpha_{1}$, there exists a quadratic extension $F / k$, unramified at the places of $S_{0}$ and all the places of bad reduction of $A$, such that

$$
\begin{aligned}
\operatorname{Sel}_{2}\left(A_{1}^{F}\right) & \subset \operatorname{Sel}_{2}\left(A_{1}\right), \\
\alpha_{1} & \in \operatorname{Sel}_{2}\left(A_{1}^{F}\right), \\
\beta & \notin \operatorname{Sel}_{2}\left(A_{1}^{F}\right), \\
\operatorname{Sel}_{2}\left(A_{i}^{F}\right) & =\operatorname{Sel}_{2}\left(A_{i}\right) \quad \text { for } i \neq 1 .
\end{aligned}
$$

Proof. Let $F=k(\sqrt{q})$ be as in Lemma 7.1. Let $i \in\{1, \ldots, r\}$. Since $F$ is split at each $v \in S$, we have $A_{i}^{F} \times_{k} k_{v} \cong A_{i} \times_{k} k_{v}$, so that the Selmer conditions at $S$ are identical for $A_{i}$ and $A_{i}^{F}$. These conditions are also identical for all primes where both $A_{i}$ and $A_{i}^{F}$ have good reduction, and this includes the primes $w_{j}$ if $j \neq i$. At $w_{i}$ the extension $F / k$ is unramified, and by condition (f) we can apply Lemma 4.2, so we obtain $\delta\left(A_{i}\left(k_{w_{i}}\right)\right)=\delta^{F}\left(A_{i}^{F}\left(k_{w_{i}}\right)\right)$.

It remains to check the behaviour at $\mathfrak{q}$, which is a prime of good reduction for $A_{i}$. If $i \neq 1$ then Frob $\mathfrak{q}_{\mathfrak{q}}=h$, and from condition (d) and formula (7) we deduce $\mathrm{H}^{1}\left(k_{\mathfrak{q}}, A_{i}[2]\right)=0$, so the Selmer conditions for both $A_{i}$ and $A_{i}^{F}$ at $\mathfrak{q}$ are vacuous. This proves that $\operatorname{Sel}_{2}\left(A_{i}^{F}\right)=\operatorname{Sel}_{2}\left(A_{i}\right)$ whenever $i \neq 1$.

In the rest of the proof we work with $A_{1}$. The Selmer conditions for $A_{1}$ and $A_{1}^{F}$ are the same at each place $v \neq \mathfrak{q}$. Thus $\operatorname{loc}_{\mathfrak{q}}\left(\alpha_{1}\right)=0$ implies $\alpha_{1} \in \operatorname{Sel}_{2}\left(A_{1}^{F}\right)$. Moreover, $\delta\left(A_{1}\left(k_{\mathfrak{q}}\right)\right) \cap \delta^{F}\left(A_{1}^{F}\left(k_{\mathfrak{q}}\right)\right)=0$ by Lemma 4.3. By property (5) in Lemma 7.1 we have $\operatorname{loc}_{\mathfrak{q}}(\beta) \neq 0$, so we conclude that $\beta \notin \operatorname{Sel}_{2}\left(A_{1}^{F}\right)$.

To prove that $\operatorname{Sel}_{2}\left(A_{1}^{F}\right) \subset \operatorname{Sel}_{2}\left(A_{1}\right)$, it is enough to show that for $A_{1}$ the Selmer condition at $\mathfrak{q}$ is implied by the Selmer conditions at the other places of $k$. Indeed, let $\xi \in \mathrm{H}^{1}\left(k, A_{1}[2]\right)$ be an element satisfying the Selmer condition at each place $v \neq \mathfrak{q}$, but not necessarily at $\mathfrak{q}$. By global reciprocity the $\operatorname{sum}_{\operatorname{of}} \operatorname{inv}_{v}(\beta \cup \xi) \in \frac{1}{2} \mathbb{Z} / \mathbb{Z}$ over all places of $k$, including the Archimedean places, is 0 . Since the images of $\xi$ and $\beta$ in $\mathrm{H}^{1}\left(k_{v}, A_{1}[2]\right)$ belong to $\delta\left(A_{1}\left(k_{v}\right)\right)$ for all $v \neq \mathfrak{q}$, we obtain $\operatorname{inv}_{v}(\beta \cup \xi)=0$. By the global reciprocity we $\operatorname{deduce} \operatorname{inv}_{\mathfrak{q}}(\beta \cup \xi)=0$. The nonzero element $\operatorname{loc}_{\mathfrak{q}}(\beta)$ generates $\delta\left(A_{1}\left(k_{\mathfrak{q}}\right)\right)$, because

$$
\delta\left(A_{1}\left(k_{\mathfrak{q}}\right)\right)=A_{1}[2] /\left(\operatorname{Frob}_{q}-1\right)=A_{1}[2] /(g-1)=\mathbb{Z} / 2,
$$

where we used (6) and the fact that Frob $\mathfrak{q}_{\mathfrak{q}}$ acts on $A_{1}[2]$ as the element $g$ of condition (c). Since $A_{1}$ is principally polarised, $\delta\left(A_{1}\left(k_{\mathfrak{q}}\right)\right)$ is a maximal isotropic subspace of $\mathrm{H}^{1}\left(k_{\mathfrak{q}}, A_{1}[2]\right)$ (see the beginning of Section 4). Therefore, $\operatorname{inv}_{\mathfrak{q}}(\beta \cup \xi)=0$ implies that the image of $\xi$ in $\mathrm{H}^{1}\left(k_{\mathfrak{q}}, A_{1}[2]\right)$ lies in $\delta\left(A_{1}\left(k_{\mathfrak{q}}\right)\right)$.

End of proof of Theorem 2.3. The extension $F / k$ is unramified at all the places where $A$ has bad reduction, so replacing $A$ by $A^{F}$ preserves condition (f) for each $A_{i}$. Conditions (a), (b), (c), (d), (e) and (g) are not affected by quadratic twisting. By 
repeated applications of Proposition 7.2 we can find a quadratic extension $F / k$ such that $\alpha_{i}$ is the only nonzero element in $\operatorname{Sel}_{2}\left(A_{i}^{F}\right)$, for all $i=1, \ldots, r$. The exact sequence (10) for $A_{i}^{F}$ shows that $\amalg\left(A_{i}^{F}\right)[2]$ is of size at most 2. If the 2-primary subgroup of $\amalg\left(A_{i}^{F}\right)$ is finite, then, by Proposition 5.2, the number of elements in $\amalg\left(A_{i}^{F}\right)[2]$ is a square. Thus $\amalg\left(A_{i}^{F}\right)[2]=0$, so that the image of $\alpha_{i}$ in $\mathrm{H}^{1}\left(k, A_{i}^{F}\right)$ is 0 . Then the image of $\alpha$ in $\mathrm{H}^{1}\left(k, A^{F}\right)$ is 0 , so that $Y^{F} \cong A^{F}$ and hence $Y^{F}(k) \neq \varnothing$. This implies that $\tilde{Y}^{F}(k) \neq \varnothing$ and hence $X=\tilde{Y} / \iota_{\tilde{Y}}$ has a $k$-point.

It remains to prove that $k$-points are Zariski dense in $X$. Since $Y^{F}(k) \neq \varnothing$, we have $Y^{F} \simeq A^{F}$, so we may identify $X$ with $\operatorname{Kum}\left(A^{F}\right)$. Hence it will suffice to show that $A^{F}(k)$ is Zariski dense in $A^{F}$. For each $i$, the exact sequence (10) for $A_{i}^{F}$ shows that $A_{i}^{F}(k) / 2 \neq 0$. Since $A_{i}^{F}[2](k)=0$ by condition (a), we see that $A_{i}^{F}(k)$ is infinite. The neutral connected component of the Zariski closure of $A_{i}^{F}(k)$ in $A_{i}^{F}$ is an abelian subvariety $B \subset A_{i}^{F}$ of positive dimension. By condition (a) we must have $B=A_{i}^{F}$. Thus the set $A_{i}^{F}(k)$ is Zariski dense in $A_{i}^{F}$ for each $i=1, \ldots, r$, so that $A^{F}(k)$ is Zariski dense in $A^{F}$.

Proof of Proposition 1.1. For each $i=1, \ldots, n$, we have the exact sequence

$$
0 \longrightarrow E_{i}(\mathbb{Q}) / 2 \longrightarrow \mathrm{H}^{1}\left(\mathbb{Q}, E_{i}[2]\right) \longrightarrow \mathrm{H}^{1}\left(\mathbb{Q}, E_{i}\right)[2] \longrightarrow 0 .
$$

By assumption there is a class $\alpha_{i} \in \mathrm{H}^{1}\left(\mathbb{Q}, E_{i}[2]\right)$ that goes to the class of the torsor $Y_{i}$ in $\mathrm{H}^{1}\left(\mathbb{Q}, E_{i}\right)[2]$. The restriction of $\alpha_{i}$ to $\mathrm{H}^{1}\left(\mathbb{R}, E_{i}[2]\right)$ is nonzero, hence $\alpha_{i} \neq 0$. Recall from Section 6 that the fixed-point set of the antipodal involution $\iota_{Y}$ on $Y=\prod_{i=1}^{n} Y_{i}$ is $Z=\prod_{i=1}^{n} Z_{i}$, where $Z_{i}$ is a torsor for $E_{i}$ [2] defined by $\alpha_{i}$.

Consider the double covering of smooth projective varieties $\pi: \tilde{Y} \rightarrow X=\tilde{Y} / \iota_{\tilde{Y}}$ whose branch locus is $Z \times_{k} \mathbb{P}_{k}^{n-1} \subset X$. Let $V \subset X$ be the complement to the branch locus, and let $U=\pi^{-1}(V)$. Then $\pi: U \rightarrow V$ is a torsor with the structure group $\mu_{2}$.

We need to show that a real point $M \in X(\mathbb{R})$ path-connected with a rational point $P \in X(\mathbb{Q})$ can be approximated by a point in $X(\mathbb{Q})$. In our assumptions we have $Z(\mathbb{R})=\varnothing$, hence $P \in V(\mathbb{Q}), M \in V(\mathbb{R})$ and the path connecting $P$ and $M$ is contained in $V(\mathbb{R})$. There exists a unique extension $F$ of $\mathbb{Q}$ of degree $[F: \mathbb{Q}] \leq 2$ such that $P$ lifts to a $\mathbb{Q}$-point $\tilde{P}$ on the quadratic twist $U^{F}$. Moreover, $M$ lifts to an $\mathbb{R}$-point $\tilde{M}$ in $U^{F}$ which is path connected with $\tilde{P}$.

We note that $U^{F}$ is naturally a subset of the quadratic twist $Y^{F}=\prod_{i=1}^{n} Y_{i}^{F}$. Recall from the introduction that each $Y_{i}^{F}$ is a torsor for $E_{i}^{F}$ defined by the image of $\alpha_{i}$ under the map

$$
\mathrm{H}^{1}\left(\mathbb{Q}, E_{i}[2]\right)=\mathrm{H}^{1}\left(\mathbb{Q}, E_{i}^{F}[2]\right) \longrightarrow \mathrm{H}^{1}\left(\mathbb{Q}, E_{i}^{F}\right) .
$$

Now $U^{F}(\mathbb{Q}) \neq \varnothing$ implies $Y^{F}(\mathbb{Q}) \neq \varnothing$, thus $Y^{F}$ is a trivial torsor, i.e., $Y^{F} \cong \prod_{i=1}^{n} E_{i}^{F}$. Therefore, $\alpha_{i}$ goes to zero in $\mathrm{H}^{1}\left(\mathbb{Q}, E_{i}^{F}\right)$, so $\alpha_{i}$ is a nonzero element of $E_{i}^{F}(\mathbb{Q}) / 2$. Thus $E_{i}^{F}$ has a $\mathbb{Q}$-point not divisible by 2 . By assumption $E_{i}^{F}[2](\mathbb{Q})=E_{i}[2](\mathbb{Q})=0$, 
so this point has infinite order in $E_{i}^{F}$. It follows that $\mathbb{Q}$-points of $E_{i}^{F}$ are dense in the neutral connected component of $E_{i}^{F}(\mathbb{R})$ for each $i=1, \ldots, n$. But we have $Y^{F} \cong \prod_{i=1}^{n} E_{i}^{F}$, so $\mathbb{Q}$-points are dense in the connected component of $Y^{F}(\mathbb{R})$ containing $\tilde{P}$ and $\tilde{M}$. Hence we can find a $\mathbb{Q}$-point on $X=\operatorname{Kum}(Y)=\operatorname{Kum}\left(Y^{F}\right)$ which is as close as we wish to $M$.

\section{Acknowledgements}

While working on this paper Harpaz was supported by the Fondation Sciences Mathématiques de Paris. The paper was finalised when Skorobogatov was at the Institute for Advanced Study in Princeton where he was supported by the Charles Simonyi Endowment. We are grateful to Jean-Louis Colliot-Thélène, Tim Dokchitser, Evis Ieronymou, and René Pannekoek for helpful discussions and to the referee for a careful reading and detailed comments.

\section{References}

[Bhargava et al. 2014] M. Bhargava, C. Skinner, and W. Zhang, “A majority of elliptic curves over $\mathbb{Q}$ satisfy the Birch and Swinnerton-Dyer conjecture", preprint, 2014. arXiv 1407.1826

[Bosch et al. 1990] S. Bosch, W. Lütkebohmert, and M. Raynaud, Néron models, Results in Mathematics and Related Areas (3) 21, Springer, Berlin, 1990. MR 1045822 Zbl 0705.14001

[Bright et al. 2016] M. Bright, T. D. Browning, and D. Loughran, "Failures of weak approximation in families", Compos. Math. (online publication April 2016), 1-41.

[Burnside 1911] W. Burnside, Theory of groups of finite order, 2nd ed., Cambridge University Press, 1911. MR 0069818 Zbl 42.0151 .02

[Cohen 1979] S. D. Cohen, "The distribution of the Galois groups of integral polynomials", Illinois J. Math. 23:1 (1979), 135-152. MR 516576 Zbl 0402.12005

[Colliot-Thélène and Skorobogatov 2000] J.-L. Colliot-Thélène and A. N. Skorobogatov, "Descent on fibrations over $\mathbf{P}_{k}^{1}$ revisited", Math. Proc. Cambridge Philos. Soc. 128:3 (2000), 383-393. MR 1744112 Zbl 1024.14003

[Colliot-Thélène et al. 1998] J.-L. Colliot-Thélène, A. N. Skorobogatov, and P. Swinnerton-Dyer, "Hasse principle for pencils of curves of genus one whose Jacobians have rational 2-division points", Invent. Math. 134:3 (1998), 579-650. MR 1660925 Zbl 0924.14011

[Dokchitser and Dokchitser 2011] T. Dokchitser and V. Dokchitser, "Root numbers and parity of ranks of elliptic curves”, J. Reine Angew. Math. 658 (2011), 39-64. MR 2831512 Zbl 1314.11041

[Dolgachev 2012] I. V. Dolgachev, Classical algebraic geometry: A modern view, Cambridge University Press, 2012. MR 2964027 Zbl 1252.14001

[Gonzalez-Dorrego 1994] M. R. Gonzalez-Dorrego, “(16, 6) configurations and geometry of Kummer

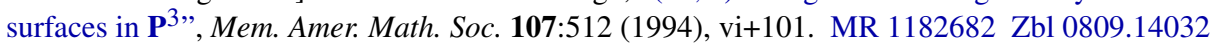

[Hall 2011] C. Hall, "An open-image theorem for a general class of abelian varieties", Bull. Lond. Math. Soc. 43:4 (2011), 703-711. With an appendix by E. Kowalski. MR 2820155 Zbl 1225.11083

[Harpaz and Wittenberg 2016] Y. Harpaz and O. Wittenberg, "On the fibration method for zero-cycles and rational points”, Ann. of Math. (2) 183:1 (2016), 229-295. MR 3432584 Zbl 06541586 
[Holmes and Pannekoek 2015] D. Holmes and R. Pannekoek, "The Brauer-Manin obstruction on Kummer varieties and ranks of twists of abelian varieties”, Bull. Lond. Math. Soc. 47:4 (2015), 565-574. MR 3375924 Zbl 1326.11032

[Klagsbrun 2012a] Z. Klagsbrun, "Elliptic curves with a lower bound on 2-Selmer ranks of quadratic twists”, Math. Res. Lett. 19:5 (2012), 1137-1143. MR 3039836 Zbl 1285.11085

[Klagsbrun 2012b] Z. Klagsbrun, "Selmer ranks of quadratic twists of elliptic curves with partial rational two-torsion", preprint, 2012. arXiv 1201.5408

[Kramer 1981] K. Kramer, "Arithmetic of elliptic curves upon quadratic extension", Trans. Amer. Math. Soc. 264:1 (1981), 121-135. MR 597871 Zbl 0471.14020

[Kuwata and Wang 1993] M. Kuwata and L. Wang, "Topology of rational points on isotrivial elliptic surfaces”, Internat. Math. Res. Notices 4 (1993), 113-123. MR 1214702 Zbl 0804.14008

[Liu 1996] Q. Liu, "Modèles entiers des courbes hyperelliptiques sur un corps de valuation discrète", Trans. Amer. Math. Soc. 348:11 (1996), 4577-4610. MR 1363944 Zbl 0926.11043

[Mazur 1972] B. Mazur, "Rational points of abelian varieties with values in towers of number fields", Invent. Math. 18 (1972), 183-266. MR 0444670 Zbl 0245.14015

[Mazur 1992] B. Mazur, "The topology of rational points", Experiment. Math. 1:1 (1992), 35-45. MR 1181085 Zbl 0784.14012

[Mazur 1995] B. Mazur, "Speculations about the topology of rational points: an update", pp. 165182 in Columbia University Number Theory Seminar (New York, 1992), Astérisque 228, Société Mathématique de France, Paris, 1995. MR 1330932 Zbl 0851.14009

[Mazur and Rubin 2007] B. Mazur and K. Rubin, "Finding large Selmer rank via an arithmetic theory of local constants", Ann. of Math. (2) 166:2 (2007), 579-612. MR 2373150 Zbl 1219.11084

[Mazur and Rubin 2010] B. Mazur and K. Rubin, "Ranks of twists of elliptic curves and Hilbert's tenth problem”, Invent. Math. 181:3 (2010), 541-575. MR 2660452 Zbl 1227.11075

[Milne 1986] J. S. Milne, Arithmetic duality theorems, Perspectives in Mathematics 1, Academic Press, Boston, 1986. MR 881804 Zbl 0613.14019

[Polishchuk 2003] A. Polishchuk, Abelian varieties, theta functions and the Fourier transform, Cambridge Tracts in Mathematics 153, Cambridge University Press, 2003. MR 1987784 Zbl 1018.14016

[Poonen and Rains 2011] B. Poonen and E. Rains, "Self cup products and the theta characteristic torsor”, Math. Res. Lett. 18:6 (2011), 1305-1318. MR 2915483 Zbl 1297.18005

[Poonen and Rains 2012] B. Poonen and E. Rains, "Random maximal isotropic subspaces and Selmer groups”, J. Amer. Math. Soc. 25:1 (2012), 245-269. MR 2833483 Zbl 1294.11097

[Poonen and Stoll 1999] B. Poonen and M. Stoll, "The Cassels-Tate pairing on polarized abelian varieties”, Ann. of Math. (2) 150:3 (1999), 1109-1149. MR 1740984 Zbl 1024.11040

[Sah 1977] C. H. Sah, "Cohomology of split group extensions, II”, J. Algebra 45:1 (1977), 17-68. MR 0463264 Zbl 0352.18021

[Skorobogatov 2001] A. Skorobogatov, Torsors and rational points, Cambridge Tracts in Mathematics 144, Cambridge University Press, 2001. MR 1845760 Zbl 0972.14015

[Skorobogatov 2009] A. Skorobogatov, "Diagonal quartic surfaces", (2009). Abstract from Belabas et al., "Explicit methods in number theory", Oberwolfach Rep. 6:3 (2009), 1843-1920. MR 2664991 Zbl 1192.00048

[Skorobogatov 2010] A. Skorobogatov, "del Pezzo surfaces of degree 4 and their relation to Kummer surfaces”, Enseign. Math. (2) 56:1-2 (2010), 73-85. MR 2674855 Zbl 1198.14035 
[Skorobogatov and Swinnerton-Dyer 2005] A. Skorobogatov and P. Swinnerton-Dyer, "2-descent on elliptic curves and rational points on certain Kummer surfaces", Adv. Math. 198:2 (2005), 448-483. MR 2183385 Zbl 1085.14021

[Skorobogatov and Zarhin 2008] A. N. Skorobogatov and Y. G. Zarhin, "A finiteness theorem for the Brauer group of abelian varieties and K3 surfaces", J. Algebraic Geom. 17:3 (2008), 481-502. MR 2395136 Zbl 1157.14008

[Skorobogatov and Zarhin 2012] A. N. Skorobogatov and Y. G. Zarhin, "The Brauer group of Kummer surfaces and torsion of elliptic curves", J. Reine Angew. Math. 666 (2012), 115-140. MR 2920883 Zbl 1256.14036

[Swinnerton-Dyer 2000] P. Swinnerton-Dyer, "Arithmetic of diagonal quartic surfaces, II", Proc. London Math. Soc. (3) 80:3 (2000), 513-544. MR 1744774 Zbl 1066.11029

[Swinnerton-Dyer 2001] P. Swinnerton-Dyer, "The solubility of diagonal cubic surfaces", Ann. Sci. École Norm. Sup. (4) 34:6 (2001), 891-912. MR 1872424 Zbl 1003.11028

[Wisbauer 1991] R. Wisbauer, Foundations of module and ring theory, Algebra, Logic and Applications: A handbook for study and research 3, Gordon and Breach Science Publishers, Philadelphia, PA, 1991. MR 1144522 Zbl 0746.16001

[Wittenberg 2007] O. Wittenberg, Intersections de deux quadriques et pinceaux de courbes de genre 1/Intersections of two quadrics and pencils of curves of genus 1, Lecture Notes in Mathematics 1901, Springer, Berlin, 2007. MR 2307807 Zbl 1122.14001

Communicated by Jean-Louis Colliot-Thélène Received 2015-05-08 Revised 2016-02-08 Accepted 2016-03-12

harpaz@dma.ens.fr Département de Mathématiques et Applications, École Normale Supérieure, 45 rue d'Ulm, Paris 75005, France

a.skorobogatov@imperial.ac.uk Department of Mathematics, Imperial College, South Kensington Campus, London, SW7 2BZ, United Kingdom Institute for Information Transmission Problems, Russian Academy of Sciences, 19 Bolshoi Karetnyi, Moscow, 127994, Russia 


\section{Algebra \& Number Theory}

msp.org/ant

\section{EDITORS}

MANAGING EDITOR

Bjorn Poonen

Massachusetts Institute of Technology

Cambridge, USA

\author{
EDITORIAL BOARD CHAIR \\ David Eisenbud \\ University of California \\ Berkeley, USA
}

BOARD OF EDITORS

Georgia Benkart

Dave Benson

Richard E. Borcherds

John H. Coates

J-L. Colliot-Thélène

Brian D. Conrad

Hélène Esnault

Hubert Flenner

Sergey Fomin

Edward Frenkel

Andrew Granville

Joseph Gubeladze

Roger Heath-Brown

Craig Huneke

Kiran S. Kedlaya

János Kollár

Yuri Manin

Philippe Michel
University of Wisconsin, Madison, USA

University of Aberdeen, Scotland

University of California, Berkeley, USA

University of Cambridge, UK

CNRS, Université Paris-Sud, France

Stanford University, USA

Freie Universität Berlin, Germany

Ruhr-Universität, Germany

University of Michigan, USA

University of California, Berkeley, USA

Université de Montréal, Canada

San Francisco State University, USA

Oxford University, UK

University of Virginia, USA

Univ. of California, San Diego, USA

Princeton University, USA

Northwestern University, USA

École Polytechnique Fédérale de Lausanne
Susan Montgomery

Shigefumi Mori

Raman Parimala

Jonathan Pila

Anand Pillay

Victor Reiner

Peter Sarnak

Joseph H. Silverman

Michael Singer

Vasudevan Srinivas

J. Toby Stafford

Ravi Vakil

Michel van den Bergh

Marie-France Vignéras

Kei-Ichi Watanabe

Efim Zelmanov

Shou-Wu Zhang
University of Southern California, USA

RIMS, Kyoto University, Japan

Emory University, USA

University of Oxford, UK

University of Notre Dame, USA

University of Minnesota, USA

Princeton University, USA

Brown University, USA

North Carolina State University, USA

Tata Inst. of Fund. Research, India

University of Michigan, USA

Stanford University, USA

Hasselt University, Belgium

Université Paris VII, France

Nihon University, Japan

University of California, San Diego, USA

Princeton University, USA

\section{PRODUCTION}

production@msp.org

Silvio Levy, Scientific Editor

See inside back cover or msp.org/ant for submission instructions.

The subscription price for 2016 is US $\$ 290 /$ year for the electronic version, and $\$ 485 /$ year ( $+\$ 55$, if shipping outside the US) for print and electronic. Subscriptions, requests for back issues and changes of subscribers address should be sent to MSP.

Algebra \& Number Theory (ISSN 1944-7833 electronic, 1937-0652 printed) at Mathematical Sciences Publishers, 798 Evans Hall \#3840, c/o University of California, Berkeley, CA 94720-3840 is published continuously online. Periodical rate postage paid at Berkeley, CA 94704, and additional mailing offices.

ANT peer review and production are managed by EditFLOW ${ }^{\circledR}$ from MSP.

\section{PUBLISHED BY}

- mathematical sciences publishers

nonprofit scientific publishing

http://msp.org/

(C) 2016 Mathematical Sciences Publishers 


\section{Algebra \& Number Theory}

Volume $10 \quad$ No. $4 \quad 2016$

Moduli of morphisms of logarithmic schemes

JONATHAN WISE

Residual intersections and the annihilator of Koszul homologies

SEYED HAMID HaSSANZADEH and Jose NAÉLITON

The Prym map of degree-7 cyclic coverings

HERBERT LANGE and ANGELA ORTEGA

SIMON MARSHALL

Hasse principle for Kummer varieties

Yonatan HaRPaZ and AlEXEI N. SkoRobogatov

Analytic continuation on Shimura varieties with $\mu$-ordinary locus

STÉPHANE BIJAKOWSKI

A note on secondary $K$-theory

GONÇALO TABUADA

Nef cones of Hilbert schemes of points on surfaces

Barbara Bolognese, Jack Huizenga, Yinbang Lin, Eric Riedl,

BENJAMIN SCHMidT, MATTHEW WoOlF and XiaOlei ZHAO

Interpolation for restricted tangent bundles of general curves 